\title{
Fusing navigation and vision information with the transferable belief model: application to an intelligent speed limit assistant
}

\author{
Jérémie Daniel, Jean-Philippe Lauffenburger* \\ Université de Haute-Alsace (UHA) \\ Laboratoire Modélisation Intelligence Processus Systèmes (MIPS) - EA2332 \\ 12, rue des frères Lumière, 68093 Mulhouse Cedex, France
}

\begin{abstract}
The present paper focuses on the fusion, based on imprecise and uncertain information, between a Geographic Information System $(G I S)$ and a Speed Limit Sign Recognition System (SLSRS), performed on camera images. This study is dedicated to the development of a Speed Limit Assistant (SLA) in the context of vehicle driving aid. The proposed SLA is developed within the Evidence Theory framework. The information from the sources is interpreted as belief functions using a non antagonistic $b b a$ in the Transferable Belief Model (TBM) semantics. This $b b a$ ensures that the conflict which could appear after the global fusion is exclusively due to source discordances. The present paper proposes a way to manage these discordances by formalizing a conflictrelated constraint decision rule. As far as the application is concerned, a two-level (decentralized) fusion architecture is developed. The sensor relevancy is estimated in a first step, followed by the GIS intra-sensor fusion with a maximum of Credibility decision which determines the context-compliant speed candidate considering the road information given by the digital map. This allows the detection of possible errors of the GIS. The multi-sensor fusion then combines the GIS and SLSRS information considering that the sensors are independent and specialized in one speed, each. For the decision, two strategies are adopted. The first one considers the conflict as a threshold for the final speed selection, and so allows the SLA to stay undecided in case of highly conflicting situations. The second strategy employs the $5^{\text {th }}$ version of the Proportional Conflict Redistribution operator. The SLA has been tested in simulation and in real-time experiments by qualitative and quantitative performance evaluations.
\end{abstract}

Key words: Transferable Belief Model, decentralized fusion, conflict-based decision, sensor relevancy estimation.

\footnotetext{
*Corresponding author. Tel.: +33 3893369 26; fax: +33 389423282

Email addresses: jeremie.danieleuha.fr(Jérémie Daniel), jean-philippe. lauffenburger@uha.fr (Jean-Philippe Lauffenburger)
} 


\section{Introduction}

This paper focuses on an $S L A$, a system whose aim is to provide the driver with the most relevant speed limit according to the driving context. Initially, SLAs were based on a lone sensor: a camera coupled to an SLSRS. Despite large advances in SLSRS techniques, these systems are still subject to inaccuracies and false detection [1]. Another solution to determine traffic signs has emerged with the democratization of $G I S$ s based on a Global Positioning System $(G P S)$. This type of solutions investigated by [2] reveals some limitations as is the case for vision systems. Indeed, even if GISs provide much information linked to the road context, it is stored in a static digital map database. Temporary situations such as road works requiring speed limit modifications are consequently hard to detect with this sensor. To overcome both sensors' limitations, the combination of a camera and a GIS is classically done. Indeed, these two sensors are obviously complementary. The GIS gives numerous static road contextual data and the vision system gives dynamic and up to date information about the local driving context. Their combination allows the SLA to become closer to human reasoning [3]. Several techniques can be employed for their combination such as Voting techniques [4], Bayesian approaches [5], the Possibility theory [6], the Belief theory [7, 8], etc. If few studies using the Bayesian theory are proposed in the literature [9], most SLAs are based on belief functions [3, 10-12]. Indeed, belief functions constitute an intuitive, suitable framework for data imperfections (inaccuracy, incompletion, etc.) modelling and deal with source ignorance and discordance [10].

In [12], contextual information is used to estimate the reliability of the vision and navigation information. For example, in case of fog where the $S L S R S$ is subject to false detection, the vision beliefs are discounted (cf. Section 2.3.4). However, if this system correctly integrates the sensors' reliability in the fusion process, its flexibility could be improved with the consideration of additional contextual data such as those available in the GIS digital map database. [11] proposed to process the GIS information through a weighted sum of contextual criteria (urban/extra urban driving, motorway driving, etc.). These attributes, stored in the GIS database, are of great help in the description of the road context, and so allow the characterization of the confidence which can be put in the speed stored in the GIS. Nevertheless, this approach does not consider the GIS inaccuracies, such as positioning errors due to the $G P S$ and/or Dead-Reckoning $(D R)$ sensor integration, digitalization errors of the road network, etc. To overcome these limitations, [3] and [13] proposed to take account of the GIS criteria regarding their nature: several attributes inform about the relevancy of the vehicle position estimation, while others are related to the description of the static driving context. This distinction helps in the definition of the amount of confidence given to each speed candidate with respect to the GIS estimated relevancy. However, this approach is limited by the weighted sum operator for the determination of the GIS speed candidates' confidence. In addition, in [13] the belief of each GIS speed is very low even if all the criteria are coherent with the driving situation and the GIS relevancy is high. From the authors'

point of view, this may lead to a situation favouring the vision system, as was the case in [11].

This paper presents a multi-level SLA based on belief functions. The modelling 
of the data and imperfections is presented in Smets' Transferable Belief Model [14], a non-probabilistic interpretation of belief functions. In the $T B M$ in particular, the conflict generated after conjunctive combination is assigned to the empty set and no redistribution is done. Practically, this differs from most SLAs which consider the closed world assumption. This choice will be further discussed in section 2.3.7.

The first fusion level, consisting in the local association of the GIS data, is based on a multi-criterion aggregation for the determination of reliable navigation information. The second level performs a global association by fusing the information from both sensors (GIS and SLSRS) to define the final speed limit and its level of confidence. An important contribution corresponds to the conjunctive combination of several criteria of the GIS to provide the best speed information with respect to all candidates. The benefits of the multi-criterion fusion consist in the estimation of the GIS relevancy in the basic belief assignment ( $b b a$ ) using the GPS dilution of precision, the map-matching performance and the accuracy of the map. In addition, the local association helps to detect the GIS false information and to determine the appropriate navigation speed more efficiently using a local decision step. Indeed, the navigation system stores speed limit data in the database independently of the criteria describing the road situation. The multi-criterion fusion, performed for each speed candidate, helps to define their respective confidence and can therefore select the one which best suits the situation detected. Due to the $b b a$ model, no conflict can be generated during this association, allowing non-ambiguous decision making. Without the local decision, the SLA would select the common hypothesis between the GIS and the vision system, even if it is not coherent with the situation [15].

In the multi-sensor fusion, each source is considered as specialized and independent. The non-overlapping $b b a[16]$ defining the belief in a speed, its contrary and the ignorance, ensures that the potential conflict is exclusively related to the source discordance. The conflict which may appear during the global association is used for the decision as in [17] or [18]. By formalizing a decision criterion explicitly integrating the conflict, the present work proposes a way to manage multi-sensor discordance. This allows the $S L A$ to remain undecided in case of highly conflicting situations. On the other hand, another speed (conflict redistributed speed) is selected after a conflict redistribution performed with the Proportional Conflict Redistribution operator 5 (PCR5).

The work presented here extends previous papers $[15,19,20]$ by providing a study of the conflict management strategies and a conflict-based decision rule in the multisensor context. Based on real experiments, a qualitative and quantitative evaluation of the system performance is given.

This paper is organized as follows: Section 2 summarizes the basics of belief functions and provides a discussion about conflict management mechanisms. Section 3 focuses on the approach adopted to combine information from the navigation and vision systems. Section 4 describes the mathematical apparatuses used to determine the best speed limit. Section 5 presents the results of this $S L A$ while section 6 concludes this paper. 


\section{Theoretical Background}

\subsection{Evidence Theory Basics}

The Evidence Theory, also known as Dempster-Shafer Theory (DST), has been mathematically formalized by Shafer [8] based on the seminal work of Dempster [7]. This theory models the belief level in an event through functions defined on subsets (singletons and/or unions) to model pieces of information provided either by objective sources (such as sensors) or subjective ones (such as human experts). This section presents the main aspects of $D S T$ required for the present $S L A$. Some knowledge about the DST is assumed.

Consider a problem for which all the discrete solutions (also called hypotheses) $H_{j}, j=1,2, \ldots, K$, with $K$ the number of possible hypotheses, define the frame of discernment $\Theta$ :

$$
\Theta=\left\{H_{1}, H_{2}, \ldots, H_{K}\right\}=\bigcup_{j=1}^{K}\left\{H_{j}\right\}
$$

$\Theta$ is the ignorance, i.e. the union of all hypotheses. Its corresponding referential subset, a power set denoted $2^{\Theta}$ of $2^{K}$ disjunctions of $H_{j}$ is such that:

$$
2^{\Theta}=\left\{\emptyset,\left\{H_{1}\right\}, \ldots,\left\{H_{K}\right\},\left\{H_{1}, H_{2}\right\}, \ldots,\left\{H_{2}, H_{3}\right\}, \ldots,\left\{H_{1}, H_{2}, H_{3}\right\}, \ldots, \Theta\right\}
$$

$\emptyset$ represents the impossible hypothesis. For practical reasons, the notation $H_{i} \cup H_{j}$ will be preferred to $\left\{H_{i}, H_{j}\right\}$ in the following parts of the paper. The veracity of a proposition $A$ of $2^{\Theta}$ is characterized by its basic belief mass ( $\mathrm{bbm}$ ) or mass $m$ defined as follows:

$$
\begin{aligned}
m: \quad & 2^{\Theta} \rightarrow[0,1] \\
& A \mapsto m(A), \sum_{A \in 2^{\Theta}} m(A)=1
\end{aligned}
$$

In opposition to the probability theory, the veracity is defined on all disjunctions of $2^{\Theta}$. Several definitions concerning $\mathrm{bbm}$ can be found in [21].

\subsection{Specialized Sources and Non-overlapping bba Model}

The basic belief assignment ( $b b a$ ) consists in the definition of the bbm for each proposition of $2^{\Theta}$. The procedure mainly depends on the problem considered. In the present study, specialized sources are used [22]. A specialized source gives information about one specific hypothesis $\left(H_{j}\right)$ of $\Theta$ by defining a $b b a$ on $H_{j}, \overline{H_{j}}$ and $\Theta$. Note that $\overline{H_{j}}$ corresponds to the disjunction of all the hypotheses of $\Theta$ different from $H_{j}$ so that

$$
\overline{H_{j}}=\left\{H_{1} \cup H_{2} \cup \cdots \cup H_{j-1} \cup H_{j+1} \cup \cdots \cup H_{k}\right\}=\Theta \backslash\left\{H_{j}\right\}
$$

Different analytic models characterizing these bbas are available. In [23, 24], respectively cosine and exponential functions are adopted. Among the different mathematical models, the one initiated by Rombaut [16] and enhanced by Royère [25] is 


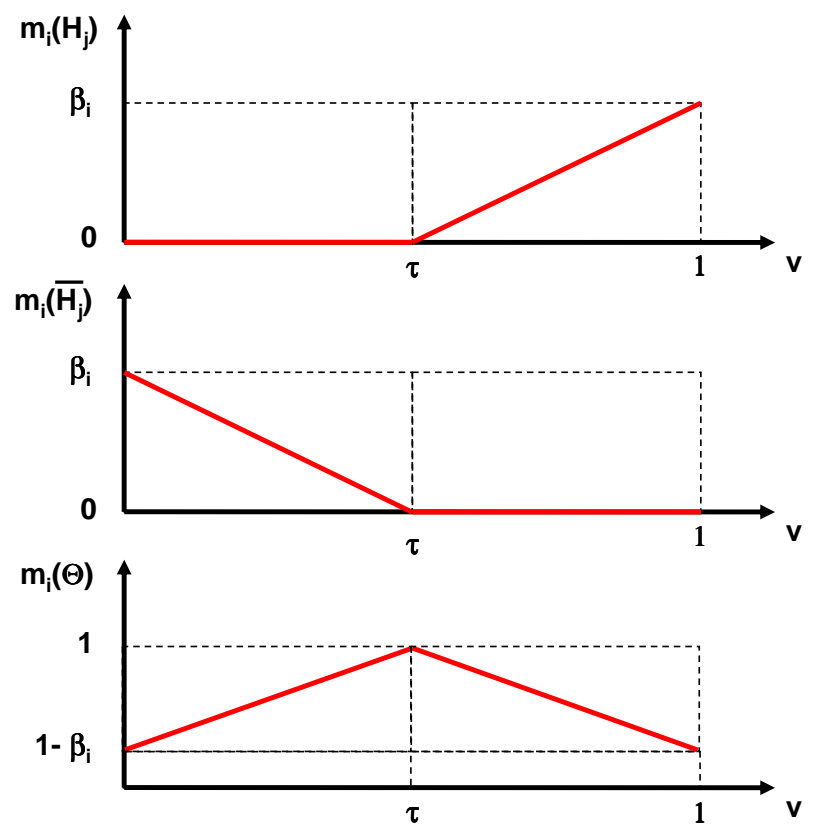

Figure 1: Basic Belief Assignment Model [16]

particularly interesting. It is based on a non-antagonistic condition so that the simultaneous generation of a $\mathrm{bbm}$ on $H_{j}$ and $\overline{H_{j}}$ is not possible. A representation of this model, for any source $i$, is shown in Fig. 1 and has the following analytic expression:

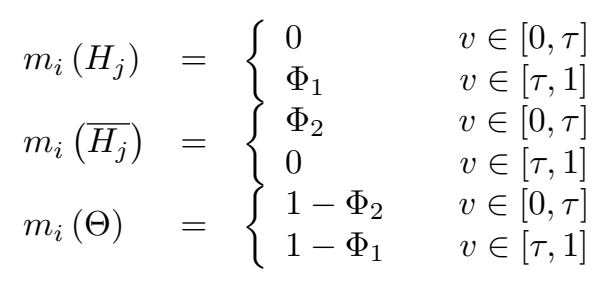

This model depends on four parameters:

- The variable $v$, which usually refers to a measurement from the considered information source, gathers the belief amount which can be given to a sensor information;

- $\Phi_{1}$ and $\Phi_{2}$ describe the evolution of the $b b a$ with respect to $v$. As mentioned earlier, different solutions such as linear or trigonometric expressions, quadratic polynomials, Gaussian curves, etc. can be used;

- $\tau$ defines the behaviour of the model, i.e. the transition between the belief in $H_{j}$ and $\overline{H_{j}}$. An optimistic/pessimistic estimation is obtained respectively with $\tau<0.5$ and $\tau>0.5$; the model is neutral if $\tau=0.5$; 
- $\beta_{i}$ defines the maximum mass value which can be allocated to $H_{j}$ and $\overline{H_{j}}$.

\subsection{Information Combination and Conflict Management}

\subsubsection{Dempster Combination Rule}

Combination gathers the different information of the sources. The choice of the combination operator must be made wisely, as it has a direct impact on the fusion results. This particular point will be illustrated in section 5 devoted to the experimental results. The most straightforward combination operator is the conjunctive operator $\cap$ [21]. By satisfying the associativity as well as commutativity properties, a fusion order definition over the $p$ combined sources is not required:

$$
m_{1 \ldots p}^{\cap}(A)=\sum_{A_{1} \cap \ldots \cap A_{p}=A} \prod_{j=1}^{p} m_{j}\left(A_{j}\right)
$$

When disjunctions with an empty intersection $\left(A_{i} \cap A_{j}=\emptyset\right)$ are combined, a mass $m(\emptyset)$ is generated. Usually, it is interpreted as the conflict between sources. Under Shafer's hypotheses of exhaustivity of $\Theta$ and exclusivity of the propositions $H_{j}$, $m(\emptyset)=0$. That is why Shafer proposes to normalize the combined masses defining the orthogonal sum operator also known as the Dempster operator $\oplus$ :

$$
\begin{aligned}
& \left\{\begin{array}{l}
m_{1 \ldots p}^{\oplus}(A)=\kappa \cdot m_{1 \ldots p}^{\cap}(A) \\
m_{1 \ldots p}^{\oplus}(\emptyset)=0
\end{array}\right. \\
& \text { with }: \kappa=\frac{1}{1-m_{1 \ldots p}^{\cap}(\emptyset)}=\frac{1}{1-\sum_{A_{1} \cap \ldots \cap A_{p}=\emptyset} \prod_{j=1}^{p} m_{j}\left(A_{j}\right)}
\end{aligned}
$$

This operator presents the advantage of being associative and commutative. Nevertheless, normalization redistributes the conflict equally on all the proposition masses whatever the conflict level. This limitation has been clearly shown by Zadeh in his well known counter-intuitive example [26], and largely discussed by several authors such as Wilson [27] or more recently Haenni [28]. Discussions about conflict origins lead to the definition of numerous combination rules [25, 29-35]. An extensive review of some of them can be found in [36] while a large discussion about their applicability and their axiomatic justifications is available in [21].

\subsubsection{Conflict Origins and Management Solutions}

The belief mass $m(\emptyset)$ can be of different origins ${ }^{1}$ and so, due to the non exhaustiveness of the discernment frame, to a source unreliability, or to the discordance of the source information. Finally, the non-idempotence of some operators also leads to pieces of conflict. Its particularity is to be absorbing, i.e. it tends to increase with the number of combinations [37]. Its management can be performed in four different ways:

- Considering a different modelling framework (cf. Section 2.3.3);

\footnotetext{
${ }^{1}$ and it could sometimes be difficult to distinguish the contribution of each of them.
} 
- Discounting the bbas to take account of the a priori known sources' reliability (cf. Section 2.3.4);

- Combining via specific operators (cf. Section 2.3.5);

- Using the conflict as a piece of information in the decision making (cf. Section 2.3.6).

The next sections describe these four approaches and focus on the conflict management methods and operators used for the SLA.

\subsubsection{Opening the World}

To define an exhaustive, exclusive discernment frame (known as closed world with $m(\emptyset)=0)$ is rather difficult for real applications. Consequently, two other modelling frameworks have been suggested: the open world from Smets [14] and the extended open world [16, 25, 38]. In Smets' TBM, $\Theta$ is defined as the container of all known hypotheses but not all possible ones ( $\Theta$ is exclusive but not exhaustive) so that as a mass $m(\emptyset)>0$ is possible. This is assumed for the $S L A$. Under the assumption of reliable sources, this non-zero mass can only be due to the non-exhaustiveness of $\Theta$. In this particular case, $\emptyset$ represents a reject class describing the unknown hypotheses. Smets conjunctive operator is such that:

$$
\left\{\begin{array}{l}
m_{1 \ldots p}^{S}(A)=m_{1 \ldots p}^{\cap}(A) \\
m_{1 \ldots p}^{S}(\emptyset)=m_{1 \ldots p}^{\cap}(\emptyset) .
\end{array}\right.
$$

The conflict related to the non-exhaustivity of the discernment frame can be treated by adding to $\Theta$ an alternative hypothesis $*$ representing all the unknown propositions not explicitly defined in $\Theta[16,25,38]$. This singleton allows the new discernment frame $\Theta^{*}$ to become exhaustive $\left(\Theta^{*}=\Theta \cup\{*\}\right)$. A non zero value of the $m(\emptyset)$ is then only linked to the sources' unreliability or to their discordance. In [21], Smets raises the problem of considering this extended open world, especially when decision is necessary.

\subsubsection{Discounting}

The conflict can be generated by a source error or false measurement, i.e. by a nonfully reliable source. The consideration of the reliability of a source $i$ can therefore avoid the generation of conflict not related to the discordance between sources. To take account of the source reliability, DST introduces discounting factors [8] used to lower the $b b a$ of a hypothesis so that:

$$
\left\{\begin{array}{l}
m_{i}^{\prime}(A)=\alpha_{i} \cdot m_{i}(A) \\
m_{i}^{\prime}(\Theta)=1-\alpha_{i}\left(1-m_{i}(\Theta)\right)
\end{array}\right.
$$

with $A \neq \emptyset$ and $\alpha_{i} \in[0,1]$ the reliability of source $i$. This solution reduces the masses given by the source considered over proposition $A$ and increases the mass of the ignorance. When a source is completely unreliable $\left(\alpha_{i}=0\right)$, the discounted belief function $m_{i}^{\prime}$ defines the total ignorance $\left(m_{i}^{\prime}(\Theta)=1\right)$ which is the neutral element of 
Dempster's orthogonal sum. The definition of $\alpha_{i}$ has been largely studied by Yager in decision making within an uncertainty framework $[39,40]$. It has been shown that, in addition to the consideration of the source reliability, $\alpha_{i}$ can also be determined according to subjective elements such as expert Strength Of Belief (SOB). The discounting can therefore be generalized in a more formal way with the belief function correction framework [24]. These functions can be based either on simple factors $\alpha_{i}$ or on various mathematical expressions related to different types of information ${ }^{2}$ (human expertise source reliability, etc.).

It is worth remembering that discounting is not a way to reduce the conflict but to take account of the a priori known source reliability.

\subsubsection{Conflict-related Operators}

After discounting and considering the $T B M$, the remaining conflict only refers to the source discordances. There may still be a problem when a decision must be taken as its mass is not related to any of the discernment frame hypotheses. Indeed, what should be done when there is a high conflict mass after the combination? A classic way to solve this problem is to redistribute the conflict mass. However, as mentioned in Section 2.3.1, it may lead to counter-intuitive results. To reduce these effects, numerous operators have been proposed in the literature. Yager proposed to redistribute the conflict over the ignorance [29]. But this operator is non-associative [41] implying the definition of a fusion order between the sources. In addition, the conflict is masked in the ignorance, which can be arguable in some cases ${ }^{3}$.

When sources are unreliable or give discordant propositions, disjunctive combinations can also be performed in order to lower the conflict [32]. In this case, the mass allocation is carried out on proposition unions. Conflict, resulting from an empty intersection of focal elements with conjunctive operators, can therefore not be generated. However, as this solution dilutes the information into disjunctions, it enlarges the solution set. In case of the $S L A$, the ignorance would increase with the successive combinations and finally, it would be impossible to decide about the appropriate proposition of $\Theta$. To keep the benefits of the conjunctive and disjunctive operators, Dubois and Prade [30] proposed a hybrid adaptive operator. As it avoids the generation of conflict, it is a good compromise between precision and reliability. Nevertheless, this operator is no longer associative. A close approach has been adopted for the conjunctive/disjunctive operator proposed in [25]. Contrary to Dubois and Prade's operator, the former has been shown to be associative and commutative when the bbas are based on specialized sources with the non-overlapping model (cf. Section 2.2).

In order to keep the advantage of conjunctive rules without reinforcing all the propositions when conflict is generated and redistributed, Dezert and Smarandache proposed the set of Proportional Conflict Redistribution operators (PCR) [35]. The

\footnotetext{
${ }^{2}$ The function based on a multiplication coefficient leads to the classic discounting technique.

${ }^{3}$ When evidence conflicts, it becomes difficult for the user to decide, which may lead him to be ignorant of the issue of the problem.
} 
fifth version $(P C R 5)$ presents the particular interest of performing a conflict redistribution only over the propositions generating it. For two sources, the PCR5 is defined as:

$$
\left\{\begin{array}{l}
m_{1 \ldots p}^{P C R 5}\left(A_{i}\right)=m_{1 \ldots p}^{\cap}\left(A_{i}\right)+\sum_{\substack{A_{j} \in \Theta \backslash\left\{A_{i}\right\} \\
A_{i} \cap A_{j}=\emptyset}}\left[\frac{m_{i}\left(A_{i}\right)^{2} m_{j}\left(A_{j}\right)}{m_{i}\left(A_{i}\right)+m_{j}\left(A_{j}\right)}+\frac{m_{j}\left(A_{i}\right)^{2} m_{i}\left(A_{j}\right)}{m_{j}\left(A_{i}\right)+m_{i}\left(A_{j}\right)}\right] \\
m_{1 \ldots p}^{P C R 5}(\emptyset)=0
\end{array}\right.
$$

with $m_{1 \ldots p}^{\cap}\left(A_{i}\right)$ the mass on proposition $A_{i}$ after the conjunctive combination (cf. 6 ), and $m_{1 \ldots p}^{P C R 5}\left(A_{j}\right)$ the mass on hypothesis $A_{j}$ after conflict redistribution.

Here, the redistribution is not performed over conflict-independent propositions. However the PCR5 is not associative, as for Yager's operator. Note that a generalization of the PCR5, the PCR6 has been presented in [34]. In the application considered in this paper, the conflict redistribution is performed for two sources. In this case, both PCR5 and PCR6 behave similarly [35]. An interesting approach, based on the $P C R 6$, is presented in [42]. Similarly to the approach presented in this paper, the first step in [42] consists in the conflict evaluation using a specific measure used as a weighting factor to combine the orthogonal sum results with those provided by a PCR6. The comparison of the PCR5 and the redistribution rule adopted in [42] represents an interesting topic for further researches.

\subsubsection{Conflict as Additional Information}

Considering that the frame of discernment is exhaustive and exclusive and that the reliability of the sources could be known, the conflict describes the discordance between sources. Its origins can be of different types (false detection of a source, a different interpretation, sources which are not focused on the same observation, etc.), and so can be interpreted as a piece of information during data fusion. This has been done for instance in [43] for the detection of sensor failures.

The conflict can also be characterized at different levels: auto-conflict, local conflict and global conflict [44]. If the auto-conflict is mainly used to show the absorbing property [37], the local and global conflicts could be used for clustering applications. In this type of problems, the aim is usually to determine discriminating clusters which contain elements with close characteristics. In [17], these desired features are obtained through the minimization of the intra-cluster conflicts (local conflicts) and through the maximization of the inter-cluster conflict (global conflict).

In [18], the conflict is used to match two sonar measurements. The fusion generates an amount of conflict which may vary according to the transformation used for the sonar data. The transformation generating the minimum level of conflict is selected. Finally, the conflict can guide the decision step as presented in [23]. The goal of this study is to perform data association in a Multi-Target Tracking application. When the selection of associations is performed, the conflict level of each potential association is used as a threshold which rejects the association presenting an insufficient confidence with respect to the conflict. This approach is at the origin of the decision making rule established in the SLA (cf. Section 4.3.1). 


\subsubsection{Solutions Selected for Conflict Management}

In the previous sections, it has been shown that conflict management is a wide and dynamic research area which provides various and sometimes controversial solutions [45-47]. However, a global solution for conflict management does not exist and the solution to be adopted is usually application-dependent. To help the user define the fusion strategy, Smets presented an expert system to analyze and solve the conflict [21] using the previously described solutions (discounting, open world, etc.) depending on the information available (source reliability, conflict level acceptance, etc.).

The $S L A$ described in this paper considers the $T B M$ framework in which $m(\emptyset)>0$ holds contrary to other belief functions-based $S L A$ s already mentioned in the introduction [3, 10-13]. Different reasons account for this choice. First, the non-antagonistic $b b a$ ensures that the potential conflict is only related to source information discordance, in this case, different speed limits provided by the sources. The belief mass $m_{1 \ldots p}^{S}(\emptyset)$ can then be treated as a dissimilarity measurement between the combined sources and used for the decision. Secondly, direct normalization (closed world) leads to a loss of this piece of information provided by $\emptyset$. Thirdly, in the extended open world context, the decision could be problematic if $*$ is stated to be the potential solution. In this case, using the conditioning principle, the mass from the extended open world $\Theta^{*}$ should be transferred before decision making to $\Theta$ [21], leading to the previously mentioned situation. As a consequence, only the last question raised by Smets' expert system remains: should the conflict be redistributed or not? To this question, both answers are given: first, the conflict is kept and considered in the decision making measurement. In a second approach, it is redistributed before decision making (cf. Section 4.3) using the PCR5. In this study, the conflict is used for the formalization of a decision criterion in order to guarantee a safe, reliable selection of the speed limit to be given to the driver. Finally, the sources relevancy are estimated (cf. Section 3.4) and used for definition of the bbas.

\subsection{Decision Making}

The decision consists in the selection of the most relevant solution from the discernment frame ${ }^{4}$. Selecting one solution in a set involves a loss of information (the information stored in the non-selected solutions). Decision making requires the definition of the most relevant belief measurement regarding the problem considered. The most common ones are the Credibility, Plausibility and Pignistic Probability measurements [14]. The Credibility function $(\mathrm{Cr})$ for a hypothesis $H_{j}$ is defined as:

$$
C r\left(H_{j}\right)=\sum_{A \subseteq H_{j}} m(A)
$$

This measurement is said to be pessimistic as only the propositions included in $H_{j}$ are considered. Contrary to Credibility, Plausibility $(P l)$ is an optimistic decision criterion taking account of all the propositions partially supporting $H_{j}$ :

\footnotetext{
${ }^{4}$ In rare cases, a union of hypotheses $\left(H_{i} \cup H_{j}\right)$ of the referential subset is chosen.
} 


$$
P l\left(H_{j}\right)=\sum_{A \cap H_{j} \neq \emptyset} m(A)=1-C r\left(\overline{H_{j}}\right)
$$

The Bet Probability, also called Pignistic Probability proposed by Smets [31], is a compromise between the Credibility and Plausibility measurements. It distributes, proportionally to the cardinality, the mass of a non-specific focal element among all the singletons which compose it. The use of this prudent criterion requires a combination operator which verifies the associativity property:

$$
\operatorname{BetP}\left(H_{j}\right)=\sum_{\substack{A \in 2^{\ominus} \\ H_{j} \subseteq A}} \frac{m(A)}{|A|(1-m(\emptyset))}
$$

with $|A|$ the cardinality of $A$ and $m$ a non-degenerated $\operatorname{bbm}(m(\emptyset) \neq 1)$.

In (11), (12) and (13), the decision focuses on the selection of a singleton $H_{j}$. Nevertheless, the Credibility, the Plausibility and the BetP measurements can be determined for any disjunctions of $2^{\Theta}$.

Besides the commonly employed decision measurements, several others are proposed in the literature, such as Sudano's [48], Cuzzolin's [49] or the Dezert Smarandache probabilities [50]. All of them consist in the transformation of bbms into probabilities to make a decision. In [50], Dezert and Smarandache review and compare these measurements using the Probability Information Content (PIC).

Once the measurement has been selected, the decision is made classically through the selection of the hypothesis of $\Theta$ which maximizes this probability. But other techniques are possible. The decision based on the constrained Credibility maximum presented by [51] can be cited. It consists in the selection of the hypothesis $\left(H_{j}\right)$ maximizing the Credibility function and satisfying a rejection criteria:

$$
\left\{\begin{array}{l}
C r\left(H_{\text {final }}\right)=\max _{1 \leq j \leq p} \operatorname{Cr}\left(H_{j}\right) \\
\operatorname{Cr}\left(H_{\text {final }}\right) \geq \operatorname{Cr}(A)
\end{array}\right.
$$

The proposition $A$ could be the complementary hypothesis $\overline{H_{\text {final }}}[51]$ or $\Theta$. In the open world context, $A$ can also represent the conflict mass $m(\emptyset)$.

Another interesting decision technique based on the pignistic probabilities can be risk-based decision [37]. A set of $N$ actions $\mathcal{A}=\left\{a_{1}, \ldots, a_{N}\right\}$, and a cost function $\lambda\left(a_{i} \mid H_{j}\right)$ which represents the cost induced by the selection of $a_{i}$ when the solution is $H_{j}$, are defined. The goal of the decision is then to select the least risky solution:

$$
R_{\text {Bet }}\left(H_{\text {final }}\right)=\min _{1 \leq j \leq p} \sum_{H_{j} \in \Theta} \lambda\left(a_{i} \mid H_{j}\right) \operatorname{BetP}\left(H_{j}\right)
$$

In [37], Lefevre et al. have shown that their approach can be used directly with the belief masses to determine an upper and lower limit for the risk indicator. This allows the user to define an optimistic or pessimistic decision, respectively by selecting the lower or the upper risk limit.

From a different point of view, it can be concluded that this method uses one criterion - "risk" - for decision making. Multi-criterion decision solutions are also possible, 
e.g. the Analytic Hierarchy Process $(A H P)$ presented by Saaty [52] and used for instance in [53] in the $D S m T$ framework. Several criteria with their relative importance are selected to define the most suitable proposition for the problem considered.

In the present paper, straightforward solutions are used for each of the conflict management strategies: the first decision technique (in which conflict explicitly appears in the decision rule) is derived from the constrained Credibility maximum (cf. (14)) using the conflict as a rejection criterion, while the second one (in which conflict is redistributed) relaxes the inequality in (14) and satisfies the maximum of Credibility.

\subsection{Multi-level Fusion Approach}

A basic approach to the fusion strategy would be to directly take the data of each source and fuse them together in a centralized fusion process as in [3, 11, 13]. This would help to propagate the sensor imperfections through the fusion process until the final decision. However, sources are not exempt of defaults as they may provide imperfect information, in other words, information which can be erroneous, missing, inaccurate, imprecise, etc. The source's imperfections/reliabilities can be integrated through discounting (cf. Section 2.3.4) or through the choice of specific bbas for instance. This can also be performed by a multi-level fusion architecture which allows the early detection and management of source incoherences before their combination and the final decision step. Particularly, in a context in which the discernment frame gathers numerous propositions as is the case in this application, a centralized structure would lead to the dilution of the sources' beliefs over the propositions of $2^{\Theta}$ when the relevancy of the sources is limited or when the sensors' discordance is high.

The principle of multi-level data fusion, or decentralized fusion, is to divide the global fusion process into several fusion steps with different levels of abstraction as shown in Fig. 2 [54]. This figure considers a local and a global data fusion level. The first level fuses the information obtained from sources $A$ and $B$ (respectively $S_{A}$ and $S_{B}$ ) to determine the global information $S$. These data may already be the results of a preceding fusion step. This is the case in Fig. 2, where $S_{A}$ and $S_{B}$ are determined through the local fusions of $n$ and $m$ sub-sources $S_{A n}$ and $S_{B m}$.

This structure has different advantages, such as the discrimination of information at different abstraction levels. It also allows to maintain both sensors at the same decision level in the fusion scheme, each of them providing only one candidate and the associated confidence for the fusion. Moreover, a decentralized fusion architecture may detect false source information (cf. Section 5) and enhance the performance of the global fusion. This property is essential in the highly dynamic driving context to avoid the generation of hazardous situations.

\section{Combining Visual and Navigation Information}

\subsection{Multi-level Fusion for SLAs}

The framework of the $S L A$ is depicted in Fig. 3. The first level is dedicated to the sensors' local information processing, i.e. to the treatment of the GIS and the SLSRS data independently. The speed of each sensor with its related belief mass are determined in this step. For the information provided by the camera, this corresponds to the 


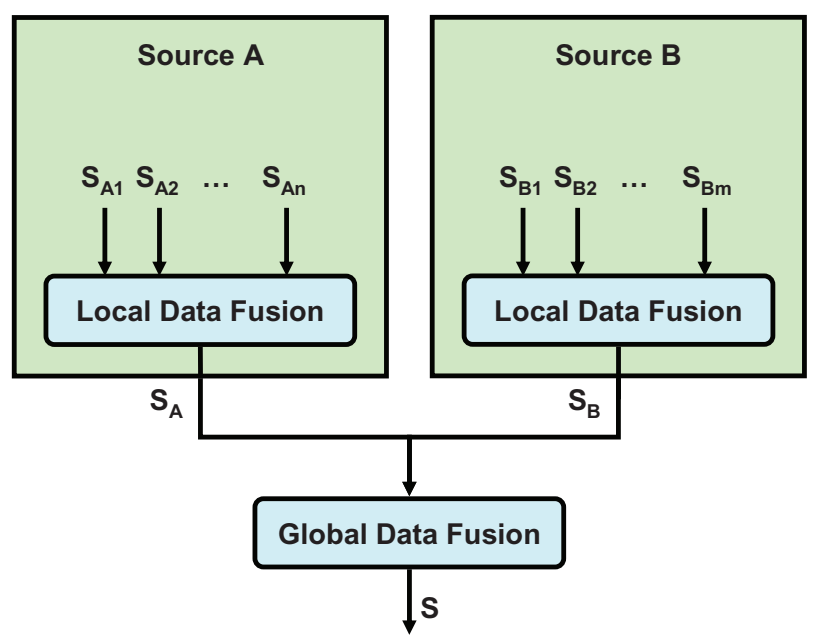

Figure 2: Multi-level Fusion Approach

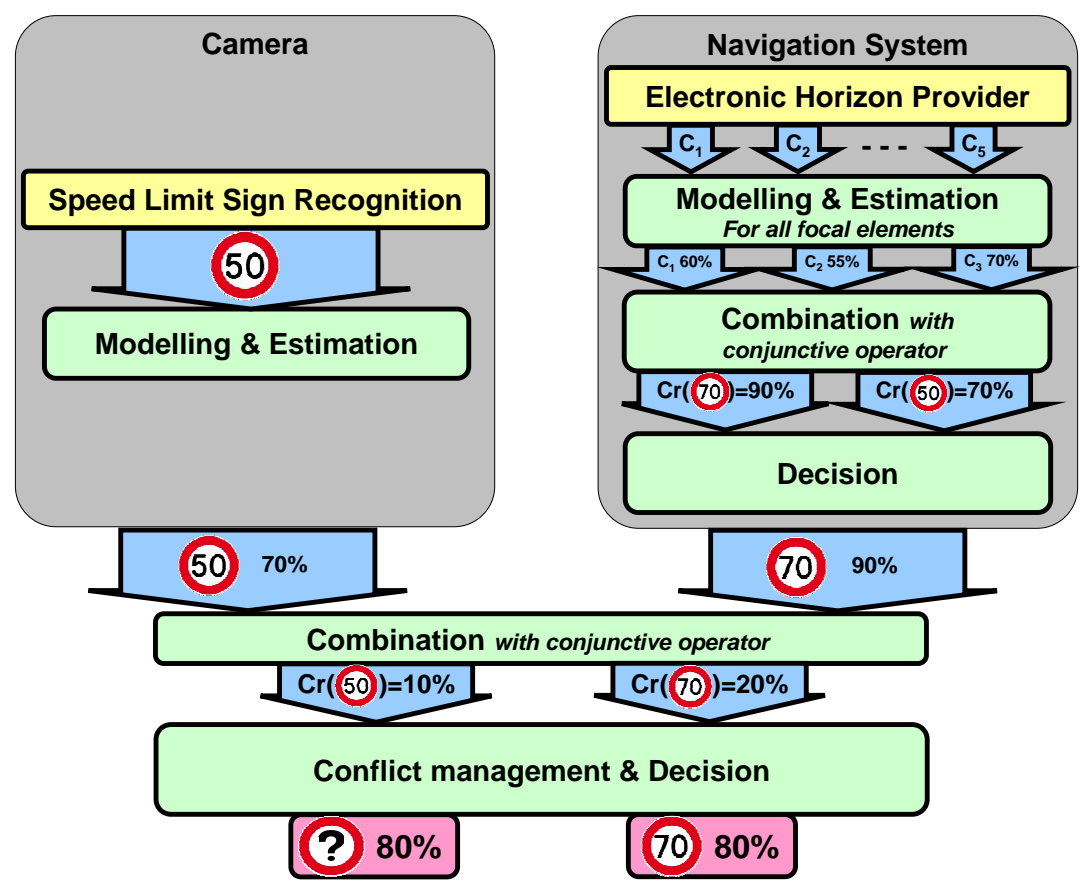

Figure 3: Multi-level Fusion Strategy 
detected speed sign and the associated detection confidence. A different approach has been adopted for the GIS information: it consists in the fusion of criteria $\left(C_{i}\right)$ corresponding to digital map attributes. These criteria describe either the road context or the GIS relevancy, and so helps to determine the speed candidates (focal set) with respect to the speed extracted from the database which is best suited for the contextual information provided by the GIS Electronic Horizon Provider (cf. Section 3.2.1). Once the speed candidates are determined, they are sequentially combined using Smets' conjunctive operator, and finally, the appropriate speed is selected regarding a local decision based on the maximum of Credibility (cf. Section 2.4). This approach helps to detect the GIS incoherences by confirming/infirming the speed extracted from the map and to avoid the propagation of unadapted speed candidates to the sensors' fusion level. Compared to [11] or [13], this solution focuses on the multi-dimensionality of the navigation information, i.e. the consideration of each criterion as an independent specialized source. In addition, the approach adopted helps to avoid a system which favours the vision information. Indeed, the removal of the local decision propagates all the navigation speed candidates, even those incoherent with the situation and characterized by small beliefs. This would lead - during the global fusion between the GIS and the vision - to an enforcement of the confidence in the common speed candidate and the selection of this proposition. This is illustrated in section 5.3.

The multi-sensor fusion is based on data provided by independent sensors specialized in one speed (the one each sensor defends). Since both sensors can be specialized for different speeds, contrary to the multi-criterion fusion, conflict can appear. Section 4.3 presents the solutions adopted to take account of the source discordance in the selection of the speed to be provided to the driver.

\subsection{GIS Description}

\subsubsection{GIS Main Components}

A GIS is composed of four main elements: a receiver, a digital map database, a map-matching algorithm and an electronic horizon provider. The receiver is dedicated to the processing of the signals from the satellite constellation (either the Global Positioning System (GPS) or the GLObal'naya NAvigatsionnaya Sputnikovaya Sistema $(G L O N A S S)$ ). This device helps to determine the user (here the vehicle) position in the Earth referential, i.e. the longitude, latitude and elevation ${ }^{5}$. This position is then eventually coupled with vehicle information (in order to improve its robustness, integrity or availability), using Dead-Reckoning ( $D R$ ) sensors. Finally, the vehicle location on the digital map database is defined. This process is performed by the map-matching algorithm which allows the determination of the electronic horizon. The latter contains the set of probable roads the vehicle is likely to take. The electronic horizon data are usable for many applications, but especially for $A D A S$ s such as the $S L A$ [55].

\subsubsection{Digital Map Database Information}

Digital map databases contain numerous geographical (point coordinates, curvatures, etc.), topological (distance between points, cover time estimation between points,

\footnotetext{
${ }^{5}$ Information about satellite configuration is also transmitted.
} 


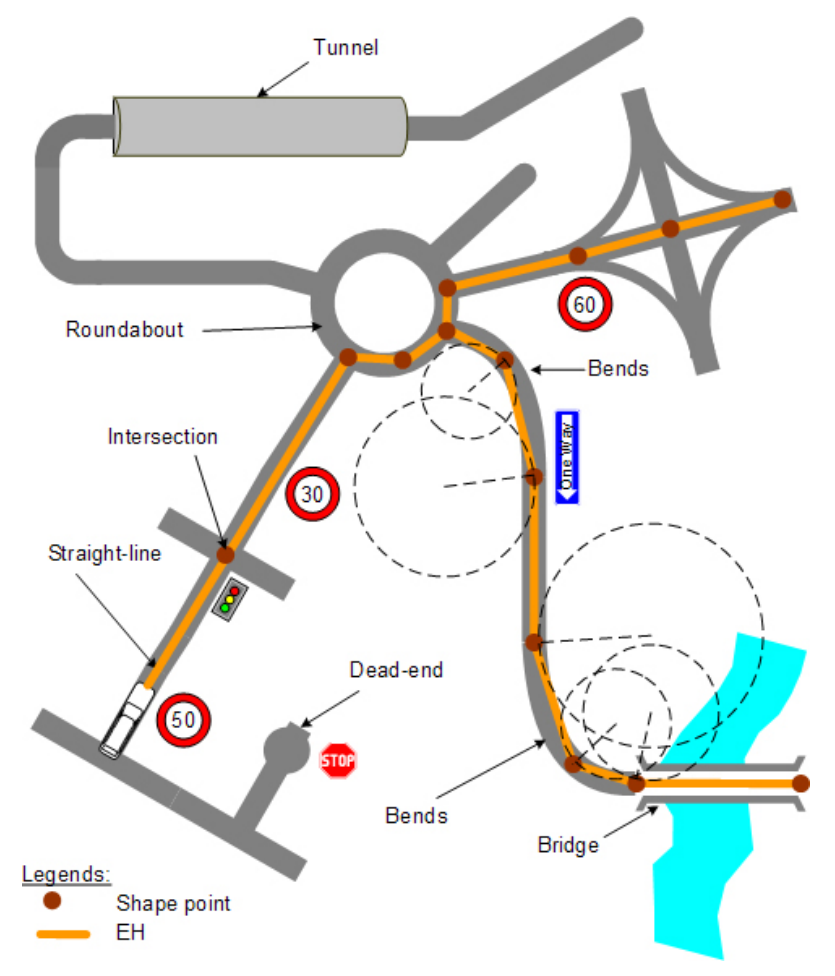

Figure 4: Example of Digital Map Database Components

etc.), and informative (traffic signs, number of lanes, etc.) attributes of the real driving infrastructure. These attributes can have different levels of abstraction; for example, the number of lanes is related to a road portion while the presence of road signs is related to specific road points. Fig. 4 presents an overview of the current database composition. The road network is vectorized based on a succession of specific points. It indeed divides roads into vectors (grey lines), also called segments, linked by nodes which usually correspond to intersections. Segments can also contain shape points (brown points) giving more precise information about the current road geometry, specific attributes, etc. Finally, this figure describes the Electronic Horizon $(E H)$ concept: it corresponds to the set of road segments the Vehicle is likely to take (yellow segments) in a fixed distance or time window.

\subsection{Selection of Relevant Navigation Criteria}

Among the information available in the GIS database, the criteria which are well suited for road context characterization have to be defined. The combination of such data (road type, city-driving status, etc.) helps to determine a belief level for each speed candidate, and thus discriminate them with respect to the encountered driving context [11]. This multi-criterion fusion approach has been enhanced by [13] which proposed a classification of the criteria into two classes. The first one describes the road context 
(road type, city driving status, etc.), and so helps to discriminate the speed candidates. On the other hand, the second refers to criteria which allow the estimation of the GIS relevancy (positioning accuracy, digital map database quality, etc.). This relevancy helps to determine the confidence which can be allocated to the GIS information. This paper follows this idea by redefining the role of each criterion. Consequently, the digital map database and localization quality attributes are used for the determination of the GIS relevancy. Contrary to the previous studies, this relevancy is used here for the definition of the bbas of five road context criteria:

- $C_{F C}$ indicates the road Functional Class, i.e. the road importance. This criterion is well adapted to speed discrimination since great importance is usually given to high speed roads (such as motorways) and inversely.

- $C_{R T}$ is related to the Road Types which are used in France: European, motorway, national, regional or local. The road type represents important information for speed discrimination. Indeed, it is related to the implicit speed limit determined in relation to the Highway code.

- $C_{C}$ describes the city-driving status. In-city and Out-of-city driving favours respectively low/high speeds.

- $C_{I}$ indicates the presence of an intersection. At crossroads, the speed limit is generally low.

- $C_{M R}$ reveals the presence of a motorway access/exit ramp. This configuration refers to particular speed limits.

The summary of the different GIS data considered and their role are presented in Table 1. A description of the GIS relevancy criteria are presented in the next section.

\subsection{Navigation Relevancy Estimation}

The authors have chosen to define the relevancy of the GIS by the estimation of the quality of three elements: positioning (which gives the raw vehicle position based on satellite signals and proprioceptive sensors), localization (which matches the vehicle position with a digitalized road), and the digital map database (which models the road network). Since the GIS performance is mainly driven by the accuracy of these three elements, an evaluation of its relevancy using their respective accuracy makes sense. These three elements are subject to inaccuracies of different origins, which may lead to false information. For this reason, it is important to define the GIS relevancy $R_{G I S}$ for the determination of the navigation belief masses. The solution adopted here integrates a relevancy estimation of the vehicle positioning, map-matching and finally the digital map level of representation:

- Vehicle positioning. The position is obtained from the fusion of GPS, odometric and proprioceptive sensors' data. The positioning accuracy is known to be variable due to several factors and especially the satellite signal transmission. The satellite geometric configuration whose quality can be given by the Geometric 
Table 1: GIS Data Summary

\begin{tabular}{|c|c|c|c|}
\hline \\
\hline Information & Name & Type & Role \\
\hline Road Importance & $C_{F C}$ & $\begin{array}{l}\text { Road context de- } \\
\text { scriber }\end{array}$ & $\begin{array}{l}\text { High importance } \\
\text { roads are charac- } \\
\text { terized by high } \\
\text { speeds }\end{array}$ \\
\hline Road Type & $C_{R T}$ & $\begin{array}{l}\text { Road context de- } \\
\text { scriber }\end{array}$ & $\begin{array}{l}\text { Implicit speeds re- } \\
\text { lated to the High- } \\
\text { way code can be } \\
\text { determined }\end{array}$ \\
\hline $\begin{array}{l}\text { City-driving } \\
\text { status }\end{array}$ & $C_{C}$ & $\begin{array}{l}\text { Road context de- } \\
\text { scriber }\end{array}$ & $\begin{array}{l}\text { In-city or Out- } \\
\text { of-city favors } \\
\text { respectively low } \\
\text { and high speeds }\end{array}$ \\
\hline Intersection status & $C_{I}$ & $\begin{array}{l}\text { Road context de- } \\
\text { scriber }\end{array}$ & $\begin{array}{l}\text { Speed is usually } \\
\text { reduced near in- } \\
\text { tersections }\end{array}$ \\
\hline $\begin{array}{l}\text { Motorway ramp } \\
\text { status }\end{array}$ & $C_{M R}$ & $\begin{array}{l}\text { Road context de- } \\
\text { scriber }\end{array}$ & $\begin{array}{l}\text { Specific speeds } \\
\text { are linked to } \\
\text { motorway ramps }\end{array}$ \\
\hline $\begin{array}{l}\text { Positioning accu- } \\
\text { racy }\end{array}$ & HDOP & $\begin{array}{l}\text { GIS quality de- } \\
\text { scriber }\end{array}$ & $\begin{array}{l}\text { Low accuracy } \\
\text { involves errors in } \\
\text { vehicle position } \\
\text { (of several me- } \\
\text { ters) which impact } \\
\text { the map-matching } \\
\text { algorithm }\end{array}$ \\
\hline $\begin{array}{l}\text { Localization } \\
\text { accuracy }\end{array}$ & MLCP & $\begin{array}{l}\text { GIS quality de- } \\
\text { scriber }\end{array}$ & $\begin{array}{l}\text { Low accuracy } \\
\text { may select the } \\
\text { wrong road in } \\
\text { the digital map } \\
\text { database }\end{array}$ \\
\hline $\begin{array}{lr}\text { Digital } & \text { Map } \\
\text { Database } & \text { Accu- } \\
\text { racy } & \end{array}$ & ADASAttribute & $\begin{array}{l}\text { GIS quality de- } \\
\text { scriber }\end{array}$ & $\begin{array}{l}\text { Low accuracy in- } \\
\text { volves a less com- } \\
\text { plete and less pre- } \\
\text { cise description of } \\
\text { the roads }\end{array}$ \\
\hline
\end{tabular}




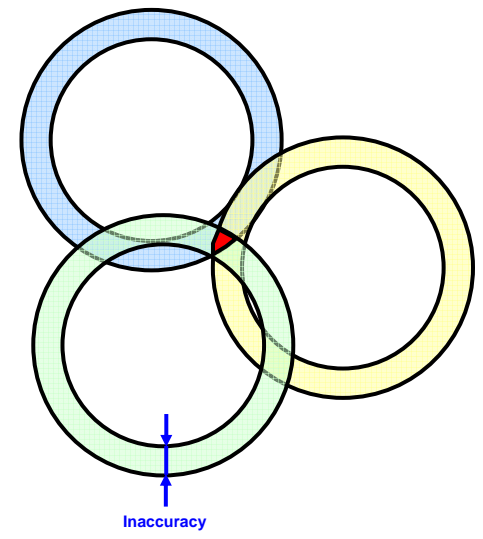

(a) Good Satellite Geometry

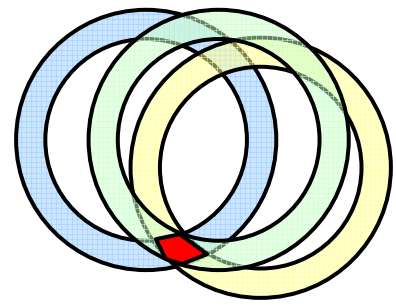

(b) Poor Satellite Geometry

Figure 5: Satellite Dilution of Precision

Dilution of Precision (GDOP) characterizing the 3D level of the GPS position [56], plays a key role in the position estimation of the receiver. The navigation system used for the real-time test presented in section 5 is composed of a 2-dimensional digital map database. That is why the paper focuses on a 2D measurement of the position precision known as the Horizontal Dilution Of Precision $(H D O P)$. In Fig. 5, the red area represents the uncertainty zone in which the vehicle is positioned based on the satellites data. A small area indicates that positioning is performed with greater accuracy. As the area size is characterized by its $H D O P$, the positioning accuracy increases with a diminution of the $H D O P$ value.

- Vehicle localization. This process determines the location of the vehicle in the digital map database with respect to the raw position, that is to say, it performs the so called map-matching . In the navigation system used, this map-matching consists in the determination of a possible vehicle location set for each vehicle position. An internal process then allocates to each candidate a probability relevant to the quality of the map-matching and called Most Likely Candidate Probability $(M L C P)$. Its value mainly depends on the current and past vehicle locations. The candidate with the best probability is then adopted as the vehicle location. Finally, as for the $H D O P$, the lower the $M L C P$, the higher the localization accuracy [57].

- Digital map database accuracy. The resolution is provided by a specific criterion named ADASAttribute which describes the quality of the road representation. This criterion is defined considering two classes of imperfections: the absolute and relative accuracies [58]. The first one refers to the error between each point coordinate of the digital map database regarding the real coordinates (Fig. 6 left plot). The second one refers to the fact that each point is correctly located 


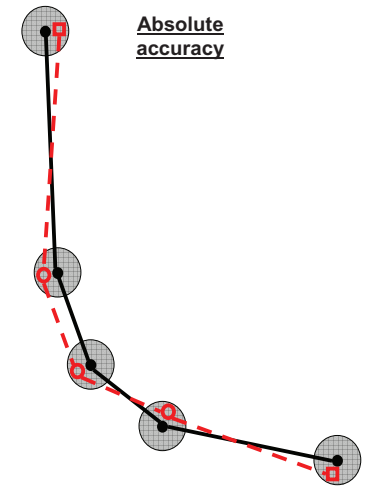

- Shape point

$5 \mathrm{~m}$ accuracy ellipse

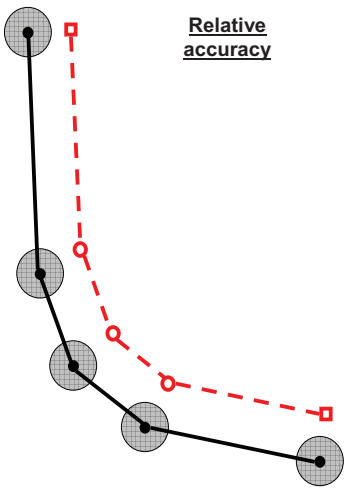

口 Database node

- Database shape point

Figure 6: Digital Map Database Inaccuracies [58]

regarding the other points of the digital map database (Fig. 6 right plot) ${ }^{6}$.

These three elements describe the quality of positioning and localization, as well as that of the digital map database, and compose the proposed navigation relevancy estimation $R_{G I S}$, as presented in (16). $R_{G I S}$ increases with the $G I S$ accuracy, e.g. low values of $H D O P$ and $M L C P$ are linked to a high confidence in the GIS data.

$$
R_{G I S}=\left(1-\left(\frac{H D O P}{H D O P_{\max }}\right)\right) \cdot\left(1-\left(\frac{M L C P}{M L C P_{\max }}\right)\right) \cdot \text { ADASAttribute }
$$

with $\frac{H D O P}{H D O P_{\max }} \in[0,1]$ and $\frac{M L C P}{M L C P_{\max }} \in[0,1]$. ADASAttribute is used as a weighting factor decreasing (or increasing) the GIS relevancy when it is (or not) activated.

\subsection{Vision Relevancy Estimation}

The vision $b b a$ is directly defined using the information provided by the SLSRS, taking account of a Forgiveness Factor $(F F)$ as presented in (17). A time-dependent coefficient which reduces the relevancy in the vision information is selected. This choice is explained by the fact that a speed sign detected several minutes before may no longer be relevant with respect to the road context. Other criteria such as distancedependent terms are less adapted to the variety and dynamics of road situations. Indeed, in urban areas, the road context dynamics can be high considering short distances, while in motorway configurations, the context evolution is limited and the travelled distances are large.

\footnotetext{
${ }^{6}$ As underlined by the reviewer, the terms absolute and relative accuracy defined by [58] respectively refer to accuracy and to precision.
} 


$$
R_{V I S}=S L S R S_{\text {confidence }}-F F(t)
$$

\subsection{Basic Belief Assignment}

Among all the possible solutions to represent knowledge using the non-antagonistic model (cf. Section 2.2 and Fig. 1), a linear evolution of the $b b a$ is considered here. The particularity of the proposed $b b a$ is to define its evolution with respect to the relevancies of the navigation $\left(R_{G I S}\right)$ and the vision $\left(R_{V I S}\right)$ systems respectively described by (18) and (19). This makes sense since a sensor which presents a low relevancy may provide an information unadapted to the driving situation encountered. Consequently, contrary to the use of the discounting (reducing the belief mass in a proposition by increasing the ignorance), the present approach allocates mass to the ignorance and to the speed complementary. This solution is more adapted to the $A D A S$ domain which requires a high level of safety.

$$
\begin{aligned}
& m_{i, j}\left(H_{j}\right)= \begin{cases}0 & R_{G I S} \in[0, \tau] \\
\left(\frac{\beta_{i}}{1-\tau}\right) R_{G I S}-\frac{\beta_{i} \tau}{1-\tau} & R_{G I S} \in[\tau, 1]\end{cases} \\
& m_{i, j}\left(\overline{H_{j}}\right)= \begin{cases}-\frac{\beta_{i}}{\tau} R_{G I S}+\beta_{i} & R_{G I S} \in[0, \tau] \\
0 & R_{G I S} \in[\tau, 1]\end{cases} \\
& m_{i, j}(\Theta)= \begin{cases}\frac{\beta_{i}}{\tau} R_{G I S}+\left(1-\beta_{i}\right) & R_{G I S} \in[0, \tau] \\
-\left(\frac{\beta_{i}}{1-\tau}\right) R_{G I S}+\frac{1-\left(1-\beta_{i}\right) \tau}{1-\tau} & R_{G I S} \in[\tau, 1]\end{cases}
\end{aligned}
$$

with $m_{i, j}\left(H_{j}\right), m_{i, j}\left(\overline{H_{j}}\right)$ and $m_{i, j}(\Theta)$ respectively corresponding to the belief mass on the speed $H_{j}$, on the complementary speed $\overline{H_{j}}$ and on the ignorance $\Theta$ for criterion $i$.

Here, contrary to [23] in which the constants $\beta_{i}$ were dedicated to the $b b a$ discounting, considering the estimated source reliabilities, these terms characterize the veracity level of the association between the criteria and the speed candidates. For example, the association of a village road type with a speed of $130 \mathrm{~km} . \mathrm{h}^{-1}$ is less possible than the association of the same speed with a Motorway road type. These coefficients have been determined empirically and are complementary to the $R_{G I S}$.

$$
\begin{aligned}
& m_{v}\left(H_{v}\right)= \begin{cases}0 & R_{V I S} \in[0, \tau] \\
\left(\frac{\beta_{v}}{1-\tau}\right) R_{V I S}-\frac{\beta_{v} \tau}{1-\tau} & R_{V I S} \in[\tau, 1]\end{cases} \\
& m_{v}\left(\overline{H_{v}}\right)= \begin{cases}-\frac{\beta_{v}}{\tau} R_{V I S}+\beta_{v} & R_{V I S} \in[0, \tau] \\
0 & R_{V I S} \in[\tau, 1]\end{cases} \\
& m_{v}(\Theta)= \begin{cases}\frac{\beta_{v}}{\tau} R_{V I S}+\left(1-\beta_{v}\right) & R_{V I S} \in[0, \tau] \\
-\left(\frac{\beta_{v}}{1-\tau}\right) R_{V I S}+\frac{1-\left(1-\beta_{v}\right) \tau}{1-\tau} & R_{V I S} \in[\tau, 1]\end{cases}
\end{aligned}
$$

Coefficient $\beta_{v}$ acts as a weighting factor which modulates the impact of the SLSRS over the multi-sensor fusion. Its value has been determined empirically. 


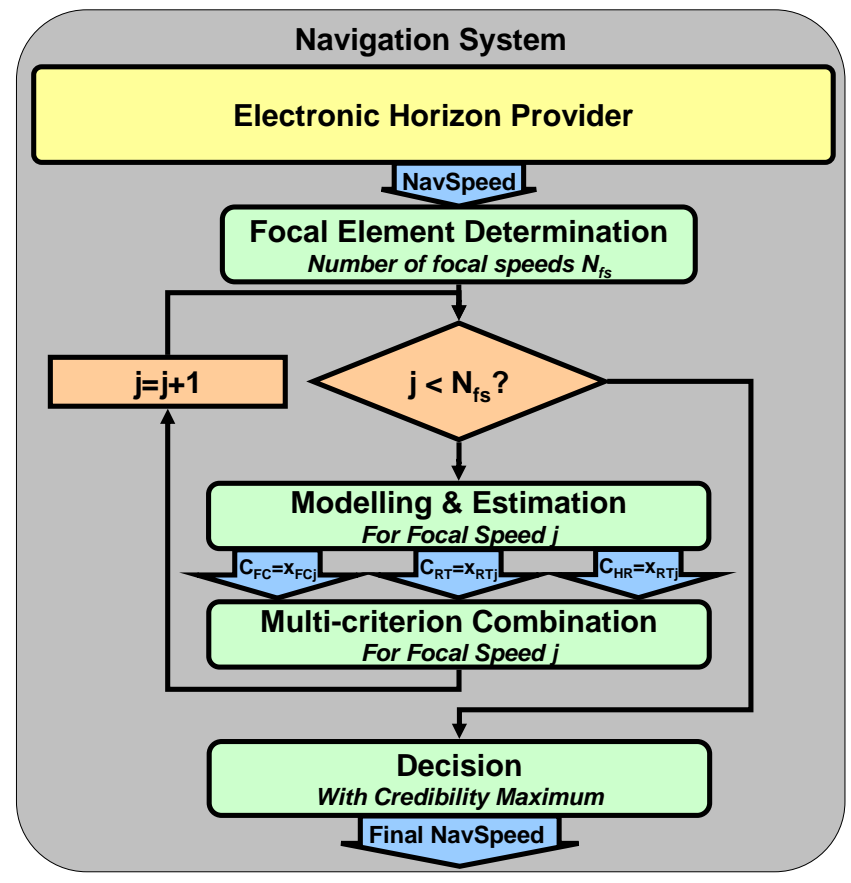

Figure 7: Multi-criterion Fusion Diagram 


\begin{tabular}{lllll}
\multicolumn{5}{c}{ Table 2: Multi-criterion Combination Table } \\
\hline & $H_{1}$ & $H_{2}$ & $\ldots$ & $H_{N_{f s}}$ \\
\hline$C_{1}$ & $m_{1,1}\left(H_{1}\right)$ & $m_{1,2}\left(H_{2}\right)$ & $\ldots$ & $m_{1, N_{f s}}\left(H_{N_{f s}}\right)$ \\
& $m_{1,1}\left(\overline{H_{1}}\right)$ & $m_{1,2}\left(\overline{H_{2}}\right)$ & & $m_{1, N_{f s}}\left(\overline{H_{N_{f s}}}\right)$ \\
& $m_{1,1}(\Theta)$ & $m_{1,2}(\Theta)$ & & $m_{1, N_{f s}}(\Theta)$ \\
\hline$C_{l}$ & $m_{l, 1}\left(H_{1}\right)$ & $m_{l, 2}\left(H_{2}\right)$ & $\ldots$ & $m_{l, N_{f s}}\left(H_{N_{f s}}\right)$ \\
& $m_{l, 1}\left(\overline{H_{1}}\right)$ & $m_{l, 2}\left(\overline{H_{2}}\right)$ & & $m_{l, N_{f s}}\left(\overline{H_{N_{f s}}}\right)$ \\
& $m_{l, 1}(\Theta)$ & $m_{l, 2}(\Theta)$ & & $m_{l, N_{f s}}(\Theta)$ \\
\hline Combination & $m_{1 \ldots l, 1}^{\cap}\left(H_{1}\right)$ & $m_{1 \ldots l, 2}^{\cap}\left(H_{2}\right)$ & $\ldots$ & $m_{1 \ldots l, N_{f s}}^{\cap}\left(H_{N_{f s}}\right)$ \\
Results & $m_{1 \ldots l, 1}^{\cap}\left(\overline{H_{1}}\right)$ & $m_{1 \ldots l, 2}^{\cap}\left(\overline{H_{2}}\right)$ & & $m_{1 \ldots l, N_{f s}}^{\cap}\left(\overline{H_{N_{f s}}}\right)$ \\
& $m_{1 \ldots l, 1}^{\cap}(\Theta)$ & $m_{1 \ldots l, 2}^{\cap}(\Theta)$ & & $m_{1 \ldots l, N_{f s}}^{\cap}(\Theta)$ \\
& $m_{1 \ldots l, 1}^{\cap}(\emptyset)$ & $m_{1 \ldots l, 2}^{\cap}(\emptyset)$ & & $m_{1 \ldots l, N_{f s}}^{\cap}(\emptyset)$ \\
\hline
\end{tabular}

\section{Speed Limit Definition by Multi-level Fusion}

\subsection{GIS Local Association}

The multi-criterion fusion is performed following the strategy presented in Fig. 7. Each GIS speed involves the definition of $N_{f s}$ focal elements. The criteria bbas are defined sequentially for each of these candidates, using the model presented in section 3.6. By interpreting the criteria as specialized sources providing opinions on the situation, pieces of information defining the driving context are generated and combined in the TBM context. This is based on the distinction between sensors and information sources made for instance in [12]. In this study, the navigation belief masses are discounted by vision information sources without creating any a priori dependence between both sensors when they are finally combined. Here, the sensor (the $G I S$ ) provides several information sources (the criteria) independently of each other. The common discernment frame $\Theta=\left\{H_{1}, H_{2}, \ldots, H_{k}\right\}$ and the power set $2^{\Theta}$ are specialized for a specific speed such as $\Theta=\left\{H_{j}, \overline{H_{j}}\right\}$ and $2^{\Theta}=\left\{\emptyset, H_{j}, \overline{H_{j}}, \Theta\right\}$. The multi-criterion fusion is performed using Smets' conjunctive operator (cf. (8)) for $l$ criteria over the $N_{f s}$ propositions. Table 2 describes how the local and partial knowledge represented by each criterion $b b a\left(\left\{m_{i, j}\left(H_{j}\right), m_{i, j}\left(\overline{H_{j}}\right), m_{i, j}(\Theta)\right\}\right)$ is combined successively for each focal speed $H_{j}$. The generalized form of this combination is given as follows $[25]^{7}$ :

\footnotetext{
${ }^{7}$ Remember that, formally, Smets' operator $m_{1 \ldots p}^{S}()$ is equivalent to the conjunctive operator $m_{1 \ldots p}^{\cap}()$.
} 
Table 3: Multi-sensor Combination Table

\begin{tabular}{|c|c|c|c|}
\hline & & & Multi-sensor Combination Results \\
\hline \multirow{9}{*}{ 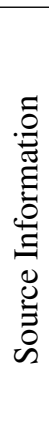 } & & $m_{1}\left(H_{1}\right)$ & \multirow{9}{*}{$\begin{array}{c}m_{1 \ldots p}^{\cap}(\emptyset) \\
m_{1 \ldots p}^{\cap \ldots p}\left(H_{1}\right) \\
\ldots \\
m_{1 \ldots p}^{\cap}\left(H_{p}\right) \\
m_{1 \ldots p}^{\cap}\left(H_{1} \cup H_{2}\right) \\
\ldots \\
m_{1 \ldots p}^{\cap}\left(H_{s} \cup \ldots \cup H_{l}\right)\end{array}$} \\
\hline & $S_{1}$ & $m_{1}\left(\overline{H_{1}}\right)$ & \\
\hline & & $m_{1}(\Theta)$ & \\
\hline & & $m_{2}\left(H_{2}\right)$ & \\
\hline & $S_{2}$ & $m_{2}\left(\overline{H_{2}}\right)$ & \\
\hline & & $m_{2}(\Theta)$ & \\
\hline & & $\vdots$ & \\
\hline & & $m_{p}\left(\underline{H_{p}}\right)$ & \\
\hline & $S_{p}$ & $\begin{array}{c}m_{p}\left(\overline{H_{p}}\right) \\
m_{p}(\Theta)\end{array}$ & \\
\hline
\end{tabular}

$$
\begin{aligned}
& m_{1 \ldots l, j}^{\cap}\left(H_{j}\right)=\prod_{i=1}^{l}\left(1-m_{i, j}\left(\overline{H_{j}}\right)\right)-\prod_{i=1}^{l} m_{i, j}(\Theta) \\
& m_{1 \ldots l, j}^{\cap}\left(\overline{H_{j}}\right)=\prod_{i=1}^{l}\left(1-m_{i, j}\left(H_{j}\right)\right)-\prod_{i=1}^{l} m_{i, j}(\Theta) \\
& m_{1 \ldots l, j}^{\cap}(\Theta)=\prod_{i=1}^{l} m_{i, j}(\Theta) \\
& m_{1 \ldots l, j}^{\cap}(\emptyset)=1-\prod_{i=1}^{l}\left(1-m_{i, j}\left(H_{j}\right)\right)-\prod_{i=1}^{l}\left(1-m_{i, j}\left(\overline{H_{j}}\right)\right)+\prod_{i=1}^{l} m_{i, j}(\Theta)
\end{aligned}
$$

Since no antagonist beliefs are possible, no conflict is generated during the conjunctive combination $\left(m_{1 \ldots l, j}^{\cap}(\emptyset)=0\right)$. The final navigation speed $H_{G I S}$ chosen for the centralized fusion with the vision is selected using the Credibility maximum :

$$
H_{G I S}=\arg \max _{1 \leq j \leq N_{f s}} C r\left(H_{j}\right)=\arg \max _{1 \leq j \leq N_{f s}} m_{1 \ldots l, j}^{\cap}\left(H_{j}\right)
$$

\subsection{Multi-sensor Combination}

The GIS local decision leads to the propagation of the bba related to the adopted speed $H_{j}\left\{m_{1 \ldots l, j}^{\cap}\left(H_{j}\right), m_{1 \ldots l, j}^{\cap}\left(\overline{H_{j}}\right), m_{1 \ldots l, j}^{\cap}(\Theta)\right\}$. For the sake of clarity, this notation will be simplified hereafter, so that a source $s$ gives the mass on its speed $s$ such as $m_{s}\left(H_{s}\right)$ with $H_{s} \in \Theta$ and the global association of $p$ sensors on a speed $H_{s}$ is denoted $m_{1 \ldots p}^{\bigcap}\left(H_{s}\right)$.

Here, the combination is applied to specialized sources which may be confident in different hypotheses of $2^{\Theta}$. Each source provides a piece of information defined regarding the same $b b a$ model. The results of the multi-sensor combination are presented in Table 3 and the generalized form is given by [25]: 


$$
\begin{array}{ll}
m_{1 \ldots p}^{\cap}\left(H_{s}\right) & =m_{s}\left(H_{s}\right) \prod_{\substack{a=1 \\
a \neq s}}^{p} \gamma_{a}+m_{j}(\Theta) \prod_{\substack{a=1 \\
a \neq s}}^{p} \eta_{a} \\
m_{1 \ldots p}^{\cap}\left(H_{s} \cup H_{l}\right) & =m_{s}(\Theta) m_{l}(\Theta) \prod_{\substack{a=1 \\
a \neq s \\
a \neq l}}^{p} \eta_{a}
\end{array}
$$

and for union combinations of 2 to $\mathrm{p}-1$ hypotheses:

$$
\begin{aligned}
m_{1 \ldots p}^{\cap}\left(H_{s} \cup \ldots \cup H_{l}\right) & =m_{s}(\Theta) \ldots m_{l}(\Theta) \prod_{\substack{a=1 \\
a \neq s \\
a \neq 0}}^{p} \eta_{a} \\
m_{1 \ldots p}^{\cap}\left(\overline{H_{s}}\right) & =m_{s}\left(\overline{H_{s}}\right) \prod_{\substack{a=1 \\
a \neq j}}^{p} m_{a}(\Theta) \\
m_{1 \ldots p}^{\cap}(\Theta) & =\prod_{a=1}^{p} m_{a}(\Theta) \\
m_{1 \ldots p}^{\cap}(\emptyset) & =1-\left[\prod_{a=1}^{p} \gamma_{a}+\sum_{a=1}^{p} m_{a}\left(H_{a}\right) \prod_{\substack{b=1 \\
b \neq a}}^{p} \delta_{b}-\prod_{a=1}^{p} \eta_{a}\right]
\end{aligned}
$$

with $\gamma_{a}=\left(1-m_{a}\left(H_{a}\right)\right), \delta_{b}=\left(1-m_{b}\left(H_{b}\right)\right)$ and $\eta_{a}=\left(m_{a}\left(\overline{H_{a}}\right)\right)$

The current equations are applied to the $S L A$ combining information from $p=2$ sources, which implies the cardinality of the discernment frame $|\Theta|=2$. For the global association, the frame of discernment is then considered to be only composed of both sensor speeds. The combination equations for 2 sources which believe either in the same hypothesis or not, are presented in [19].

$$
\Theta=\left\{H_{V I S}, H_{G I S}\right\} \Rightarrow 2^{\Theta}=\left\{\emptyset, H_{V I S}, H_{G I S}, \Theta\right\}
$$

with $H_{V I S}$ the vision speed and $H_{G I S}$ the navigation speed ${ }^{8}$.

\subsection{Conflict Management and Final Decision}

As sources can give opinions over different speeds, conflict may be generated during the multi-sensor fusion. In the $T B M$ context of the $S L A$, and assuming the nonoverlapping $b b a$ model, the mass on $\emptyset$ can only be due to source discordance. Its management for the $S L A$ to provide robust and safe speed information is a contribution of this work. This can be done using several techniques (cf. Section 2.3) and according to the discussion in section 2.3.7, two strategies have been chosen. First, this paper provides a decision making solution in presence of conflict by the formalization of a selection criterion considering a conflict-related constraint. In the next sections, the

\footnotetext{
${ }^{8}$ As there are only two possible speeds in $\Theta$, the union of these speeds obviously corresponds to the ignorance $\left(H_{V I S} \cup H_{G I S}=\Theta\right)$ and $H_{V I S}=\overline{H_{G I S}}$.
} 
speed limit adopted will be called raw speed limit. Secondly, a proportional redistribution of $m(\emptyset)$ among the speed candidates with the PCR5 determines the so called conflict redistributed speed limit.

\subsubsection{Conflict as a Criterion for Decision Making}

Under the aforementioned conditions, the mass on $\emptyset$ is the consequence of the dissimilarity in the sensors' information. In critical applications such as driving safetyoriented systems, decision making in presence of (high) conflict is problematic. Neglecting the empty set belief mass by using the classic normalization is not satisfactory. At the same time, selecting a proposition with a low belief mass (for instance because of high ignorance or high conflict) is hazardous. A methodological contribution of this paper consists in the definition of a decision criterion which integrates both elements as selection constraints. The first speed information provided by the $S L A$ is consequently the speed with the Credibility maximum, considering a configurable threshold as well as the conflict level. This choice corresponds to pragmatic and safe information based on the results of the combination. If both speeds have a Credibility simultaneously below the threshold and the conflict level, no proposition will be selected:

$$
H_{\text {raw }}=\left\{\left(\arg \max _{1 \leq j \leq p} \operatorname{Cr}\left(H_{s}\right) \geq\left\{\begin{array}{l}
\text { Threshold } \\
m_{12}^{\cap}(\emptyset)
\end{array}\right), \text { undefined }\right\}\right.
$$

(24) differs from the decision strategy of [19]. In the latter, the constrained Credibility maximum with a constant threshold has been used without consideration of the conflict level.

\subsubsection{Conflict Redistributed Multi-sensor Fusion}

Because of its general redistribution of the conflict mass which may be generated by a limited number of sources over all propositions (even the ignorance is increased), the Dempster Normalization is not adapted to the current application. Here, the PCR5 operator has been selected (cf. (10) $)^{9}$. The proportional redistribution obviously increases the masses on the sensor's speeds and involves $m_{12}^{\cap}(\emptyset)=0$ without modifying the ignorance belief. Decision can then be made with the Credibility maximum:

$$
H_{\text {red }}=\arg \max _{1 \leq j \leq p} C r\left(H_{s}\right)
$$

The PCR5 is of particular interest for the given objective. A high conflict level can generally be explained by a strong belief of the sources in their respective hypothesis. The partial transfer of these beliefs to $\emptyset$ tends to reduce the combined confidence masses. The PCR5 can therefore involve a re-appearance of the strong beliefs while keeping the other combined data unchanged (ignorance, etc.).

\footnotetext{
${ }^{9}$ Note that the $P C R 5$ behaves like the $P C R 6$ for two sources.
} 


\section{Experimental Results}

This section is dedicated to the description of the results obtained using the multilevel SLA. First, the configuration of the system is given. Then, simulations and realtime experiments with the test car are presented. Some results showing the interests of the $S L A$, either for the multi-criterion or the multi-sensor fusion, have already been presented. In [20], simulations highlight the benefits in terms of speed discrimination and GIS error detection thanks to the multi-criterion fusion compared to [11]. The impact of the local decision is shown in [15]. Simulations and real-time experiments with the test car were carried out in [19] to compare two conflict management solutions. The first strategy was to select the speed with the maximum of belief considering a simple threshold constraint, while the second was to redistribute the eventual conflict using the PCR5.

The results presented in this paper fit into the continuity of those presented in the previous contributions. Indeed, the multi-criterion results of [20] are completed with a deeper analysis of the GIS relevancy impact in section 5.2. Moreover, the advantages of the focal sets definition coupled to the local decision emphasized in [15] are detailed in section 5.3. Compared to [19], this paper proposes the results obtained with the conflict-based decision criterion (cf. (24)) in order to avoid the selection of a proposition either when its belief mass is low and/or the conflict mass is high.

Finally, a quantitative analysis presents the performance rates of the SLA obtained through real experiments in section 5.4.2, and thus completes the qualitative results provided in [19] and [20].

\subsection{System Configuration}

The simulation results were obtained on MATLAB with a simulator reproducing the behaviour of the real-time SLA implemented in the test car. This simulator helps to define all the information required for the multi-level fusion: the values of the vision speed, its relevancy and the GIS parameters (HDOP, MLCP, ADASAttribute, etc.).

The test car used for real-time tests is equipped with a 12-bit grey scale ECK-100 camera from Sensata Technologies which provides VGA images $(640 * 480)$ of the vehicle front scene. The speed sign detection algorithm developed by Mines ParisTech is based on four main steps: the detection of Regions Of Interest (ROI), a segmentation of sign characters within the ROIs, a neural network-based character recognition and a classification which finally gives the detected speed limit with the confidence level in the detection. A description of this SLSRS is available in [59]. The GIS used is the Advanced Driver Assistance Systems Research Platform (ADASRP) from NAVTEQ. It is composed of the hardware and software elements of a navigation system: the GPS and vehicle signal Receiver, digital map database, map-matching algorithm and electronic horizon provider. The main advantage of ADASRP is its open access $(\mathrm{C}++$ Software Development Kit) to the electronic horizon data, and so to the criteria required for the $S L A$. A complexity measurement of the SLA is given in terms of the required computer performance and the computation load of the application. The SLSRS as well as the fusion of the GIS and the SLSRS information are implemented with the Real-Time Multi-sensor Advanced Prototyping Software (RTMAPS) developed by Intempora 
and running on a $2.13 \mathrm{GHz}$ dual core CPU and $4 G B$ RAM computer. This simple computer configuration allows the $S L A$ to work at $30 \mathrm{~Hz}$ corresponding to the camera frame rate. In these conditions, the computation load of the real-time application is around $70 \%$ for the SLSRS while the fusion represents only $20 \%$, even if a discernment frame with a high cardinality is considered (cf. (26)).

For the tests, the discernment frame considered contains all the speeds which can be given by the navigation and the vision systems. It is obviously related to speeds defined by legal driving rules, and is as follows:

$$
\Theta=\{5,10,20,30,45,50,60,70,80,90,100,110,120,130, \text { unlimited }\}
$$

Very low speeds $\left(5 \mathrm{~km} . \mathrm{h}^{-1}\right.$ to $\left.30 \mathrm{~km} . \mathrm{h}^{-1}\right)$ refer to situations which can only arise in local and specific in-city situations (car parks, school neighbourhoods, etc.) while unlimited refers to roads which do not have any speed limitation. In Europe, this mainly refers to German motorways.

For each GIS speed extracted from the digital map database, a set of focal elements is determined. These sets have been identified through real experiments performed on various road contexts. By comparing the digital map speed limit with the ground truth, the respective focal sets have been identified. As a result, a focal set consists of the GIS digital map speed and all the observed ones in case of GIS false information. The focal set table (cf. Table 4) used in this paper is adapted from the one in [11] with modifications for $70,90,110$ and $130 \mathrm{~km} \cdot \mathrm{h}^{-1}$ speeds.

\begin{tabular}{cc}
\multicolumn{2}{c}{ Table 4: Detected Speed and Associated Focal Elements } \\
\hline Navigation Speed $\left(k m \cdot h^{-1}\right)$ & Focal Elements $\left(\mathrm{km} \cdot \mathrm{h}^{-1}\right)$ \\
\hline 5 & 50 \\
10 & 50 \\
20 & 50 \\
30 & 50 \\
45 & 30,50 \\
50 & none \\
60 & $50,80,90$ \\
70 & $50,60,70,90$ \\
80 & 50,70 \\
90 & 50,70, unlimited \\
100 & $50,70,90$ \\
110 & 50,70, unlimited \\
120 & $50,70,90,110$ \\
130 & none \\
unlimited
\end{tabular}

The focal elements bbas are performed for each criterion of the GIS with a neutral ( $\tau=0.5$ ) non-antagonistic model. The GIS relevancy $R_{G I S}$ (cf. (16)) is estimated using the following parameters:

- $M L C P_{\max }=100000$, 
- $H D O P_{\max }=40$,

- ADASAttribute is equal to 0.9 or 0.7 respectively when it is/is not validated.

The $M L C P_{\max }$ and $H D O P_{\max }, A D A S A t t r i b u t e$ and $\beta_{i}$ were estimated empirically using driving records. In particular, the $M L C P_{\max }$ and $H D O P_{\max }$ correspond to the maximum values observed, while the ADASAttribute was determined considering the conservative property of equation (16). Finally, the different $\beta_{i}$ were determined by observing the occurrences of the different criterion with the ground truth.

The vision $b b a$, performed with the model presented in section 3.6, considers a Forgiveness Factor $(F F)$ which reduces the vision relevancy by $10 \%$ every $30 \mathrm{~s}$, and is thus adapted to the evolution of the road context. The vision coefficient $\beta_{v}=0.9$ has been chosen considering the optimistic property of the traffic sign detection module. Indeed, the $S L S R S$ is usually very confident in its detection ( $\geq 75 \%$ ), even in degraded conditions (partial occlusions, distance, blur, etc.).

Finally, decision making based on the conflict-related maximum of credibility (cf. Section 4.3.1) is performed with a neutral threshold of 0.5. As will be highlighted in the performance evaluation section, this is also a conservative choice since the adopted speed candidate must simultaneously satisfy:

$$
H_{\text {raw }}=\left\{\left(\arg \max _{1 \leq j \leq p} \operatorname{Cr}\left(H_{s}\right) \geq\left\{\begin{array}{l}
0.5 \\
m_{12}^{\cap}(\emptyset)
\end{array}\right), \text { undefined }\right\}\right.
$$

\subsection{Impact of the Navigation Relevancy}

This section is dedicated to the comparison of the proposed SLA results with those obtained with the weighted sum approach from [11]. Since [13] focuses on a completely different approach in the GIS information consideration, it is, in the authors' opinion, more realistic to compare their studies to [11]. Indeed, in [13], the GIS bba is performed over all discernment frame speeds considering the GIS as a unique source of information. This leads to the dilution of the GIS masses over the propositions of $2^{\Theta}$ so that the sum of masses is unitary. Consequently, small confidences are attributed to each speed. On the contrary, [11] performs a GIS bba sequentially over the different speed candidates, and so is closer to the approach adopted in this paper.

\subsubsection{Weighted Sum SLA (WS SLA)}

For comparison purposes, a brief description of [11] is given here. The latter determines the masses over the different GIS focal speeds in three steps. First, the GIS ignorance level is defined taking the number of focal elements into account (cf. (29)). Then, the $b b a$ of the speed considered is computed using a Weighted Sum (WS) according to the set of weighting coefficients presented in (28) and the ignorance (cf. (29)). Finally, the mass of $\overline{H_{j}}$ is defined so that the total mass sum equals 1.

$$
\alpha^{W S}=[0.25,0.5,0.5,1.0,1.0,0.5,1.0,0.25]
$$

In summary, the $b b a$ for the GIS speeds is expressed as: 


$$
\begin{cases}m_{G I S}^{W S}(\Theta) & =N_{f s} \cdot 0.05 \\ m_{G I S}^{W S}\left(H_{G I S}\right) & =\left(\frac{\sum_{i=1}^{8} \alpha^{W S}(i) \cdot C r i t(i)}{\sum_{i=1}^{8} \alpha^{W S}(i)}\right)-m_{G I S}^{W S}(\Theta) \\ m_{G I S}^{W S}\left(\overline{H_{G I S}}\right) & =1-m_{G I S}^{W S}\left(H_{G I S}\right)-m_{G I S}^{W S}(\Theta)\end{cases}
$$

with $N_{f s}$ the number of focal speeds and $C r i t$ the different criteria so that:

$$
\text { Crit }=\left[M L C P, A D A S \text { Attribute, } C_{F C}, C_{R T}, C_{C}, C_{I}, C_{M R}, C_{G M}\right]
$$

$C_{G M}$ is an additional criterion related to the activation of the navigation system Guidance Mode (path routing).

\subsubsection{Discussion of the Results}

In [20], two road contexts were simulated to show the interests of the multi-criterion fusion compared to [11]. The first consisted of an urban situation correctly described by the different criteria and with a GIS database speed corresponding to the ground truth. In such a configuration, both approaches were converging to the ground truth (confirming the GIS speed). Nevertheless, the paper showed a higher discriminating potential for the new $S L A$. Indeed, by generating simultaneously bbms over $H_{G I S}$, $\overline{H_{G I S}}$ and $\Theta$, the WS SLA leads to ambiguous situations in which these bbms are close. The second example of [20] focused on an incoherent criterion definition scenario with an incoherent GIS speed. Here again both systems were able to detect the GIS information incoherences, again with a larger discriminating potential for the proposed SLA which clearly rejects the wrong candidates.

It can be noted that both tests were performed with a high $G I S$ relevancy $\left(R_{G I S}=\right.$ 0.68). The question which can be raised is: what is the impact of the navigation relevancy over the multi-criterion fusion?

To show this impact, consider the driving context described in Table 5 and dealing with a situation in which the GIS provides coherent information: the navigation speed is $130 \mathrm{~km} . \mathrm{h}^{-1}$, implying the consideration of $50,70,90,110 \mathrm{~km} \cdot \mathrm{h}^{-1}$ as focal elements. The vehicle drives on a extra-urban motorway with a functional class of 0 (and so describes a high importance road) and without any intersection or motorway ramp. However, the GIS information is given considering a low relevancy $\left(R_{G I S}=0.18\right)$ due to low positioning and map-matching performance and the non validation of the ADASAttribute. Fig. 8 presents the GIS fusion results obtained with this configuration for the proposed $S L A$ and the $W S S L A$. The multi-criterion approach only generates belief masses on $\overline{H_{j}}$ and on the ignorance. This is due to the low level of the GIS relevancy variable and is coherent with the navigation configuration. This point is correctly integrated by the multi-criterion approach as it rejects the different speeds of the GIS with high beliefs (around 0.90). On the opposite, the WS SLA presents strong belief masses in the speed candidates: high speeds $\left(90 \mathrm{~km} \cdot \mathrm{h}^{-1}, 110 \mathrm{~km} \cdot \mathrm{h}^{-1}\right.$ and $130 \mathrm{~km} . \mathrm{h}^{-1}$ ) have belief masses which are higher than 0.6 showing that the weighted 
Table 5: Irrelevant Navigation Information Configuration

\begin{tabular}{|c|c|}
\hline Navigation Attributes & Interpretation \\
\hline Navigation speed $=130$ & $\overline{\mathrm{FS}}=\{50,70,90,110,130\}$ \\
\hline MLCP $=50000$ & $\mathrm{MLCP}=50000$ \\
\hline ADAS Attribute Not Validated & ADASAttribute $=0.7$ \\
\hline Functional Class $=0$ & 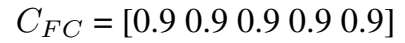 \\
\hline Motorway & 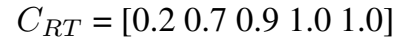 \\
\hline Out-city Driving & $C_{C}=\left[\begin{array}{lllll}0.9 & 0.5 & 0.3 & 0.3 & 0.0\end{array}\right]$ \\
\hline No Intersection & $C_{I}=\left[\begin{array}{lllll}0.3 & 0.6 & 0.8 & 0.9 & 0.9\end{array}\right]$ \\
\hline No Motorway Ramp & $C_{M R}=\left[\begin{array}{lllll}0.5 & 0.5 & 0.5 & 0.5 & 0.5\end{array}\right]$ \\
\hline $\mathrm{HDOP}=20$ & $\mathrm{HDOP}=20$ \\
\hline
\end{tabular}

sum is not able to correctly integrate the quality of the navigation information.

\subsection{Results of Local Decision and Focal Elements}

In [15], the authors presented the advantages of the local decision in the selection of the relevant GIS speed. By presenting another simulation example, this section highlights the benefits both in the focal sets and the decentralized architecture. In this example, the fusion is done over all elements of $\Theta$. Note that the different criterion values $\rho_{f s}, v_{f s}, \phi_{f s}, \psi_{f s}, \omega_{f s}$ are dependent on the focal speed considered.

A driving situation defined by a high GIS relevancy $\left(R_{G I S}=0.75\right)$, but at the same time with incoherency between the criteria and the original speed given by the GIS, is considered. Indeed, the GIS database speed is $90 \mathrm{~km} . \mathrm{h}^{-1}$ while the criteria describe an urban situation: the vehicle is on an accurately digitalized urban communal road, without any intersection nor motorway ramp. On the other hand, the vision has not correctly detected a $30 \mathrm{~km} . \mathrm{h}^{-1}$ sign and returns a speed of $130 \mathrm{~km} . \mathrm{h}^{-1}$ with a high relevancy $\left(R_{V I S}=0.75\right)$. This relevancy leads to the belief masses described in Fig. 9 top right plot which shows an average belief in the detected speed due to the neutral $b b a$ model. Note that for clarity purposes, the unlimited proposition does not appear in Fig. 9.

The GIS speed incoherency has been correctly detected by the multi-criterion fusion since it rejects the high speeds as shown in Fig. 9, top left plot. Removing the focal set does not seem to impact the multi-criterion fusion since it gives larger masses to low speeds $(>0.65)$ and provides context compliant results which infirm the GIS speed of $90 \mathrm{~km} \cdot \mathrm{h}^{-1}$.

The conflict which is generated during the combination of the GIS candidates and the vision speed is redistributed using the $P C R 5$ to provide the multi-sensor fusion results presented in Fig. 9, middle plot. The latter shows that even if the belief masses of the GIS and the SLSRS for $130 \mathrm{~km} . \mathrm{h}^{-1}$ are average (respectively 0.42 and 0.5 ) and subject to large ignorance levels, the combination leads to a high confidence in this speed (0.71). This is due to the conjunctive combination operator which favours the sources common propositions. As a consequence, the detection of the GIS error through the multi-criterion fusion is lost. 

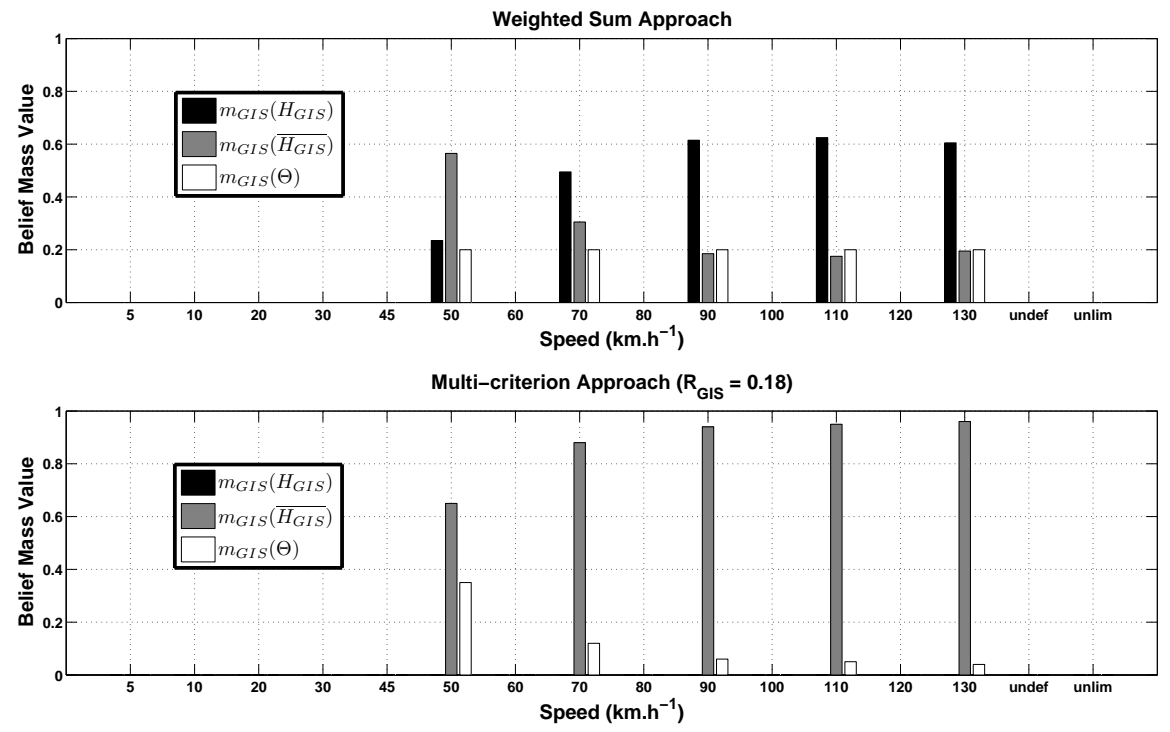

Figure 8: Multi-criterion Fusion For Irrelevant Navigation Information

Table 6: Local Decision Interest Configuration

\begin{tabular}{lc}
\hline Navigation Attributes & Interpretation \\
\hline Navigation speed $=90$ & $\mathrm{FS}=\Theta$ \\
MLCP $=15000$ & MLCP $=15000$ \\
ADAS Attribute Validated & ADASAttribute $=0.9$ \\
Functional Class $=4$ & $C_{F C}=\rho_{f s}$ \\
Communal & $C_{R T}=v_{f s}$ \\
In-city Driving & $C_{C}=\phi_{f s}$ \\
No Intersection & $C_{I}=\psi_{f s}$ \\
No Motorway Ramp & $C_{M R}=\omega_{f s}$ \\
HDOP $=1$ & $\mathrm{HDOP}=1$ \\
\hline
\end{tabular}



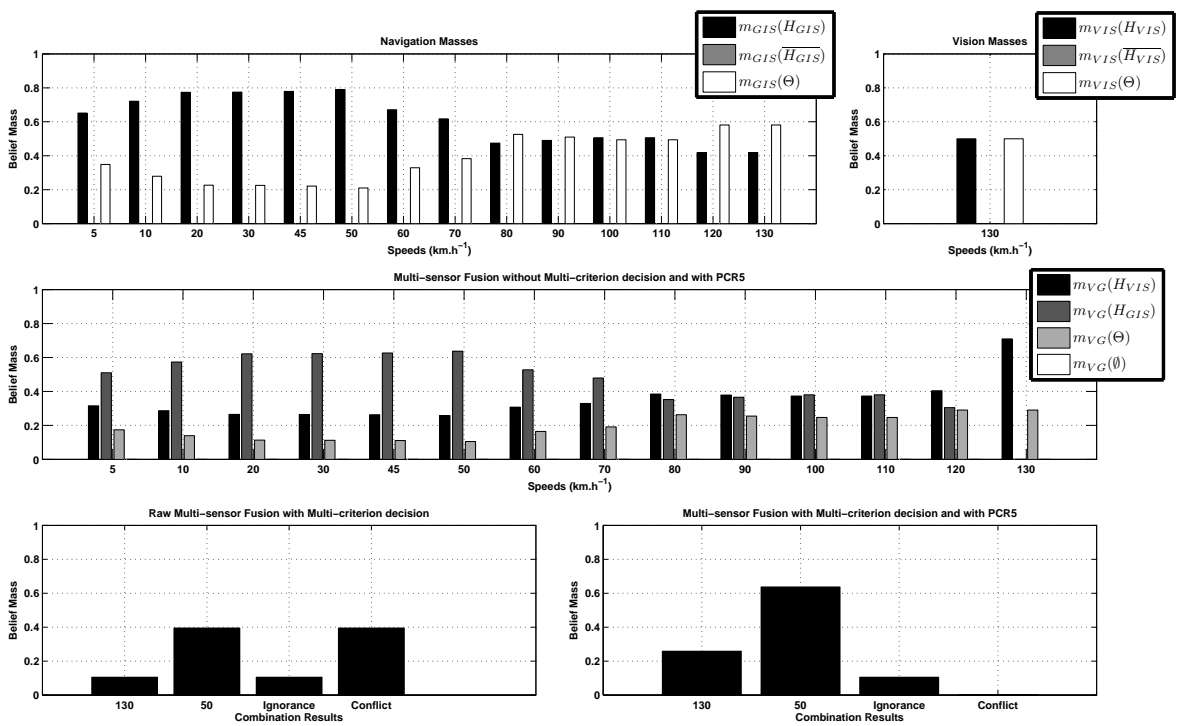

Figure 9: Multi-level Fusion Results

This limitation could be overcome by using the focal sets. Indeed, as shown in Table $4,130 \mathrm{~km} \cdot \mathrm{h}^{-1}$ is not a focal element of $90 \mathrm{~km} \cdot \mathrm{h}^{-1}$. Consequently, no masses would have been generated on $130 \mathrm{~km} . \mathrm{h}^{-1}$, and so would not have been propagated to the multi-sensor fusion. Nevertheless, the use of the focal sets is not sufficient for a general application since a change of the vision speed to $90 \mathrm{~km} . \mathrm{h}^{-1}$ (which is still incoherent with the road context) leads to the same abnormal solution.

The local decision then appears as an interesting solution. In fact, the maximum of belief after multi-criterion fusion is obtained for $50 \mathrm{~km} . \mathrm{h}^{-1}$, which would then be the GIS information adopted for multi-sensor fusion. The results of this fusion without and with conflict redistribution are respectively shown in Fig. 9, bottom left plot and bottom right plot. As the sensors are now discordant, the raw fusion obviously generates an average conflict (0.40). The combined masses are coherent with those available before the combination. The a priori information has consequently been propagated through the multi-sensor combination since the combined mass on $50 \mathrm{~km} . \mathrm{h}^{-1}$ is higher than for $130 \mathrm{~km} . \mathrm{h}^{-1}$ (respectively 0.40 and 0.11 ) and the ignorance level is low. This phenomenon is amplified with the PCR5 since their masses respectively become 0.64 and 0.26 .

\subsection{Real-time Tests}

This section is dedicated to the results obtained with the $S L A$ in real-time tests. In a first step, the qualitative results from a punctual situation previously presented in [20] are summed up in order to describe the real-time application and the $H M I$ provided in the test car. In a second step, a quantitative performance evaluation is proposed on two recorded sequences. 


\subsubsection{Qualitative Analysis}

Several qualitative studies describing the $S L A$ in real driving conditions are presented $[19,20]$. These results confirm those obtained in simulation in different conditions (concordant/discordant sensors, coherent/incoherent navigation information, etc.). They also confirm the effectiveness of the $b b a$ model, the combination and the decision strategies. In order to show the system's outputs, one of these tests is briefly summarized in this section. A snapshot of the SLA obtained in real-time using RTMAPS is shown in Fig. 10. It is divided into five parts containing (from top to bottom):

- The SLSRS speed and its associated detection confidence,

- The GIS database speed, its relevancy as well as the speed adopted after the multi-criterion fusion with its corresponding mass,

- The multi-sensor fusion results composed of the raw speed and its belief mass, the conflict and the $P C R 5$ redistributed speed with its mass,

- A graphical representation of the $S L S R S$ and multi-criterion speeds respectively on the left and right side coupled to the raw fusion speed in the centre,

- A graphical representation of the $S L S R S$ and multi-criterion speeds respectively on the left and right side coupled to the PCR5 fusion speed in the centre.

Fig. 10 was obtained near an exit of a national 3-lane expressway which is limited to $90 \mathrm{~km} . \mathrm{h}^{-1}$. The vehicle was out of city, on a road whose functional class was validated. The navigation correctly detected the expressway exit. There is consequently a slight incoherency between the navigation speed of $110 \mathrm{~km} . \mathrm{h}^{-1}$ and the road type, since French national roads are usually limited to $90 \mathrm{~km} . \mathrm{h}^{-1}$. This incoherency was detected by the multi-criterion fusion as the extracted navigation speed of $110 \mathrm{~km} . \mathrm{h}^{-1}$ was rejected and replaced by the ground truth of $90 \mathrm{~km} . \mathrm{h}^{-1}$. Furthermore, this fusion step was also able to correctly integrate the relevancy of the navigation information. Indeed, the ADASAttribute was not validated here, and thus involved an average navigation relevancy $\left(R_{G I S}=0.52\right)$. This resulted in the generation of a low belief in the navigation information (0.09). As a consequence, the multi-sensor fusion was performed over similar speeds, and so generated a high belief in $90 \mathrm{~km} \cdot \mathrm{h}^{-1}(0.59)$. Finally, as there was no conflict between sources, the conflict redistributed fusion gave the same results.

\subsubsection{Quantitative Analysis}

Two short video sequences and the corresponding GIS data extracted from the recorded test files have been selected to evaluate the $S L A$. These sequences, of nearly the same length, integrate various configurations (motorway, national road, urban, etc.) obtained in normal weather conditions (dry and sunny); their characteristics are depicted in Table 7. The first sequence mainly refers to motorway driving characterized by an average number of traffic signs and a low city proportion. Contrary to this, the second sequence presents greater road context variety: $36 \%$ of motorway and urban configuration as well as $64 \%$ of national road, described by more traffic signs. To 


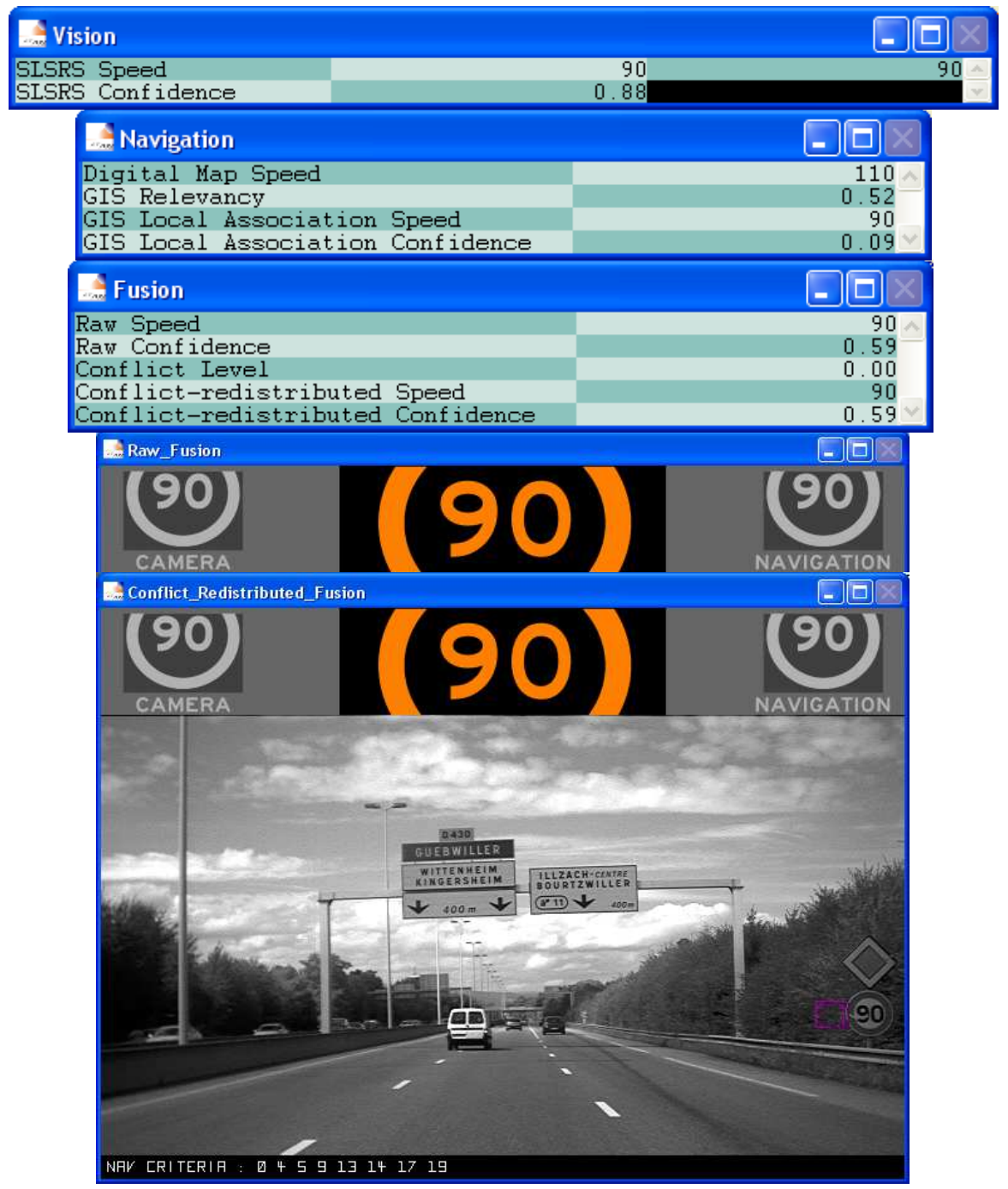

Figure 10: Real-time Fusion for Incoherent Navigation Information and with Sensors in Agreement [19] 
Table 7: Sequences Considered

\begin{tabular}{lcc}
\hline & Sequence 1 & Sequence 2 \\
\hline Duration (mm:ss) & $5: 25$ & $6: 40$ \\
Length (km) & 7.0 & 8.0 \\
Speed Sign Number & 10 & 21 \\
Road exits & 4 & 8 \\
Motorway (\%) & 80 & 36 \\
National (\%) & 20 & 64 \\
City (\%) & 11 & 36 \\
\hline
\end{tabular}

evaluate the $S L A$, a ratio of the amount of travelled time with the correct speed limit information, with respect to the total time travel, is used. The consideration of a criterion based on the travelled distance would have biased the results, especially on motorways, similarly to the vision system Forgiveness Factor (cf. Section 3.5). This evaluation consists in comparing the ground truth with the speeds returned by the different elements of the SLA:

- the vision speed directly obtained from the SLSRS,

- the speed directly extracted from the GIS database,

- the speed with the largest belief mass after multi-criterion fusion,

- the raw multi-sensor speed determined using (24) (named Raw in the Tables of results),

- the multi-sensor speed with conflict redistribution using the $P C R 5$.

The ground truth was determined manually in accordance to the road context and the Highway Code. Two strategies were considered for the performance rates computation: with and without the local decision.

Table 8 presents the results of sequence 1. It highlights that the SLSRS is efficient since it presents around $80 \%$ of correctness. On the opposite, the GIS presents surprisingly low efficiency since it gives the correct speed limit in less than 50\% of the cases. This could be explained by the areas in which these tests were carried out, namely outside large French city areas usually characterized by a high level of digitalization quality. However, if the GIS digital map speeds are not always correct, the criteria present a better representativity. This is confirmed by the multi-criterion fusion efficiency which increases the GIS efficiency by $30 \%$.

For the multi-sensor fusion, Table 8 shows that the raw fusion gives average results (around 50\%). This is due to the conservative decision rule requiring a high belief to be validated (see (27)) associated to a low conflict. As a consequence, the SLA stays regularly undecided. On the other hand, the $P C R 5$-based conflict redistribution fusion is efficient since it gives at least $80 \%$ of correctness. This fusion type also reveals the advantages of the local decision. Indeed, the PCR 5 fusion efficiency matches the vision efficiency when no local decision is applied. Contrary to this, the local decision 
Table 8: Performance Rates for Sequence 1

\begin{tabular}{lcc}
\hline SLA configuration & Without local decision $(\%)$ & With local decision $(\%)$ \\
\hline SLSRS & 80 & 80 \\
GIS & 46 & 46 \\
Multi-criterion & $60(G I S+30 \%)$ & $60(G I S+30 \%)$ \\
Raw & 51 & 48 \\
$P C R 5$ & $80(S L S R S+0 \%)$ & $86(S L S R S+8 \%)$ \\
\hline
\end{tabular}

Table 9: Performance Rates for Sequence 2

\begin{tabular}{lcc} 
& Table 9: Performance Rates for Sequence 2 \\
\hline SLA configuration & Without local decision $(\%)$ & With local decision $(\%)$ \\
\hline SLSRS & 42 & 42 \\
GIS & 39 & 39 \\
Multi-criterion & $42(G I S+8 \%)$ & $42(G I S+8 \%)$ \\
Raw & 36 & 27 \\
PCR5 & $42(S L S R S+0 \%)$ & $59(S L S R S+40 \%)$ \\
\hline
\end{tabular}

helps to propagate the multi-criterion fusion advantages to the multi-sensor fusion, thus increasing the $S L A$ efficiency up to $86 \%$.

Table 9 presents the results of the second sequence. The GIS still presents low efficiency $(39 \%)$ but, contrary to the first sequence, the SLSRS has an efficiency close to the $G I S(42 \%)$. In these degraded conditions, the advantages of the SLA are still visible. In fact, the multi-criterion fusion gives slighlty better results than the raw GIS speed, reaching the SLSRS efficiency (42\%). Then, the raw multi-sensor fusion presents low efficiency (36\% and 27\%), as it often stays in the undecided case. Nevertheless, this is a safe decision since both sensors are inconsistent. Besides, the local decision impact is clearly shown, since the PCR5 fusion efficiency matches that of the vision when the local decision is discarded. On the opposite, the local decision associated to the PCR5 increases the SLA efficiency up to $59 \%$.

\section{Conclusion}

This paper has presented a new approach to the combination of information from both a Geographic Information System $(G I S)$ and a vision system for a Speed Limit Assistant (SLA). The interpretation of the sensors' information in the Transferable Belief Model context associated to non-overlapping bbas over the propositions of the discernment frame ensure the combined mass on the empty set to be representative of source dissimilarities. This conflict can thus be used to formalize a safety-oriented decision rule for the selection of the speed limit.

First, a local GIS association, i.e. the multi-criterion fusion, is specially dedicated to the combination of intra-navigation information. Considering two types of criteria: - those characterizing the GIS relevancy and those describing the road context -, this local association defines the confidence of each speed candidate according to the road context encountered. A local decision is used to select the most relevant GIS speed which is, in a second time, combined to the vision detected speed. The inter-sensor 
fusion considers the GIS and vision as independent and specialized sources. The final speed is then selected using two strategies. On the one hand, the conflict is formally used as a threshold allowing the SLA to stay undecided when evidence conflicts. On the other hand, the conflict is redistributed using the Proportional Conflict Redistribution $(P C R)$ operator 5. This strategy helps to redistribute the conflict over the propositions generating it, providing a better view of the pieces of evidence given by each source. The proposed SLA has been evaluated in simulation as well as in real-time experiments. Qualitative and quantitative analyses show its performance compared to the ground truth.

In the future, several points may be enhanced. The local association is based on an approach which considers a multi-criterion fusion for each speed candidate successively. An improvement of the present strategy would be to consider a multi-criterion decision as in $[53,60]$ for the GIS speed determination. Finally, if the global decision strategies formally consider the conflict as additional information, decision rules which takes account of both the conflict and the ignorance level could be studied.

\section{References}

[1] D. Gavrila, Traffic sign recognition revisited, in: DAGM Symp. fur Musterekenn., Bonn, Germany, 1999, pp. 86-93.

[2] J. Ehrlich, M. Marchi, P. Jarri, L. Salesse, D. Guichon, D. Dominois, C. Leverger, LAVIA, the French ISA project: main issues and first results on technical tests, in: Intell. Trans. Syst., Madrid, Spain, 2003.

[3] A. Puthon, F. Nashashibi, B. Bradai, A complete system to determine the speed limit by fusing a GIS and a camera, in: Int. Conf. on Intell. Trans. Syst., Washington DC, USA, 2011, pp. 1686-1691.

[4] L. Lam, C. Suen, Application of majority voting to pattern recognition: An analysis of its behavior and performance, IEEE Trans. on Syst., Man, and Cybern., Part A: Syst. and Hum. 27 (1997) 553-568.

[5] E. Jaynes, Probability theory: the logic of science, Cambridge University Press, New York, 2003.

[6] L. Zadeh, Fuzzy sets, Inf. and Control 8 (1965) 338-353.

[7] A. Dempster, Upper and lower probabilities induced by a multivalued mapping, The Ann. of Math. Stat. 38 (1967) 325-339.

[8] G. Shafer, A mathematical theory of evidence, Princeton University Press, 1976.

[9] C. Bahlmann, M. Pellkofer, J. Giebel, G. Baratoff, Multi-modal speed limit assistants: Combining camera and GPS maps, in: Intell. Veh. Symp., Eindhoven, Netherlands, 2008, pp. 132-137.

[10] H. Janssen, W. Niehsen, Vehicle surround sensing based on information fusion of monocular video and digital map, in: Intell. Veh. Symp., Parma, Italy, 2004, pp. 244-249. 
[11] J.-P. Lauffenburger, B. Bradai, M. Basset, F. Nashashibi, Navigation and speed signs recognition fusion for enhanced vehicle location, in: IFAC World Congr., Seoul, South Korea, 2008, pp. 2069-2074.

[12] D. Nienhüser, T. Gumpp, J. Zöllner, A situation context aware Dempster-Shafer fusion of digital maps and a road sign recognition system, in: Intell. Veh. Symp., Xi' an, China, 2009, pp. 1401-1406.

[13] A. Puthon, F. Nashashibi, B. Bradai, Improvement of multisensor fusion in speed limit determination by quantifying navigation reliability, in: Int. Conf. on Intell. Trans. Syst., Madeira, Portugal, 2010, pp. 855-860.

[14] P. Smets, R. Kennes, The transferable belief model, Artif. Intell. 66 (1994) 191234.

[15] J. Daniel, J.-P. Lauffenburger, Multi-level Dempster-Shafer speed limit assistant, in: Int. Conf. on Belief Funct., Compiègne, France, 2012, pp. 327-334.

[16] M. Rombaut, Decision in multi-obstacle matching process using DempsterShafer's theory, in: Adv. in Veh. Control and Saf., Amiens, France, 1998, pp. $63-68$.

[17] J. Schubert, Clustering belief functions based on attracting and conflicting metalevel evidence using Potts spin mean filed theory, Inf. Fusion 5 (2004) 309-318.

[18] C. Rominger, A. Martin, Recalage et fusion d'images sonar multivues: utilisation du conflit, Revue Nationale des Technologies de l'Information E (21) (2011) 231246.

[19] J. Daniel, J.-P. Lauffenburger, Conflict management in multi-sensor DempsterShafer fusion for speed limit determination, in: Intell. Veh. Symp., Baden Baden, Germany, 2011, pp. 987-992.

[20] J. Daniel, J.-P. Lauffenburger, M. Basset, Multi-criterion Dempster-Shafer fusion for speed limit determination, in: IFAC World Congr., Milano, Italy, 2011, pp. 668-673.

[21] P. Smets, Analyzing the combination of conflicting belief functions, Inf. Fusion 8 (2007) 387-412.

[22] A. Appriou, Formulation et traitement de l'incertain et analyse multi-senseurs, in: Colloq. GRETSI, Juan-les-Pins, France, 1993, pp. 951-954.

[23] B. Mourllion, D. Gruyer, C. Royère, S. Théroude, Multi-hypotheses tracking algorithm based on the belief theory, in: Int. Conf. on Inf. Fusion, Philadelphia, USA, 2005.

[24] D. Mercier, T. Denoeux, M. Masson, Belief function correction mechanisms, Stud. in Fuzziness and Soft Comput. 249 (2010) 203-222. 
[25] C. Royère, Contribution à la résolution du conflit dans le cadre de la théorie de l'évidence : Application à la perception et à la localisation de véhicules intelligents, Ph.D. thesis, Université de Technologie de Compiègne, France (2002).

[26] L. Zadeh, On the validity of Dempster's rule of combination, memo M 79/24, Univ. of California, Berkeley (1979).

[27] N. Wilson, The assumptions behind Dempster's rule, in: Conf. on Uncertain. in Artif. Intell., San Mateo, USA, 1993, pp. 527-534.

[28] R. Haenni, Shedding new light on Zadeh's criticism of Dempster's rule of combination, in: Int. Conf. on Inf. Fusion, Philadelphia, USA, 2005, pp. 879-884.

[29] R. Yager, On the Dempster-Shafer framework and new combination rules, Inf. Sciences 41 (1987) 93-137.

[30] D. Dubois, H. Prade, Representation and combination of uncertainty with belief functions and possibility measures, Comput. Intell. 4 (1988) 244-264.

[31] P. Smets, Constructing the pignistic probability function in a context of uncertainty, Uncertain. in Artif. Intell. 5 (1990) 29-40.

[32] P. Smets, Belief functions: The disjunctive rule of combination and the generalized bayesian theorem, Int. J. of Approx. Reason. 9 (1993) 1-35.

[33] C. Murphy, Combining belief functions when evidence conflicts, Decis. Support Syst. 29 (2000) 1-9.

[34] A. Martin, C. Osswald, A new generalization of the proportional conflict redistribution rule stable in terms of decision, in: Advances and Applications of DSmT for Information Fusion: Collected Works, Vol. II, American Research Press, 2006.

[35] F. Smarandache, J. Dezert, Advances and Applications of DSmT for Information Fusion. Collected Works, American Research Press, 2009.

[36] K. Sentz, S. Ferson, Combination of evidence in Dempster-Shafer theory, Sandia report SAND2002-0835, Sandia National Laboratories (2002).

[37] E. Lefevre, O. Colot, P. Vannoorenberghe, Belief function combination and conflict management, Inf. Fusion 3 (2002) 149-162.

[38] D. Gruyer, Etude et traitement de données imparfaites pour le suivi multi-objet: application aux situations routières, Ph.D. thesis, Université de Technologie de Compiègne (1999).

[39] R. Yager, On the determination of strength of belief for decision support under uncertainty - Part i: generating strength of belief, Fuzzy Sets and Syst. 142 (2004) $117-128$. 
[40] R. Yager, On the determination of strength of belief for decision support under uncertainty - Part ii: fusing strength of belief, Fuzzy Sets and Syst. 142 (2004) $129-142$.

[41] P. Smets, The combination of evidence in the transferable belief model, IEEE Trans. on Pattern Anal. and Mach. Intell. 12 (1990) 447-458.

[42] L. Hongfei, J. Hongbin, T. Kangsheng, F. Xiaoyan, Analysis and improvement for proportional conflict redistribution rules, in: Comput. Appl. and Syst. Model., 2010.

[43] B. Marhic, L. Delahoche, C. Solau, A. Jolly-Desodt, V. Ricquebourg, An evidential approach for detection of abnormal behaviour in the presence of unreliable sensors, Inf. Fusion 13 (2012) 146-160.

[44] A. Martin, A.-L. Jousselme, C. Osswald, Conflict measure for the discounting operation on belief functions, in: Int. Conf. on Inf. Fusion, Köln, Germany, 2008, pp. $1-8$.

[45] R. Haenni, Are alternatives to Dempster's rule of combination real alternatives? comments on "about the belief function combination and the conflict management problem" - Lefevre et al., Inf. Fusion 3 (2002) 237-239.

[46] E. Lefevre, O. Colot, P. Vannoorenberghe, Reply to the comments of R. Haenni on the paper "belief function combination and conflict management", Inf. Fusion 4 (2003) 63-65.

[47] J. Dezert, W. Pei, A. Tchamova, On the validity of Dempster-Shafer theory, in: Int. Conf. on Inf. Fusion, Singapore, 2012, pp. 655-660.

[48] J. Sudano, Yet another paradigm illustrating evidence fusion, in: Int. Conf. on Inf. Fusion, Florence, Italy, 2006, pp. 1-7.

[49] F. Cuzzolin, Symbolic and Quantitative Approaches to Reasoning with Uncertainty, Vol. 5590, Springer, Berlin/Heidelberg, 2009, Ch. The intersection probability and its properties, pp. 287-298.

[50] J. Dezert, F. Smarandache, A new probabilistic transformation of belief mass assignment, in: Int. Conf. Inf. Fusion, Köln, Germany, 2008, pp. 1-8.

[51] S. Le Hegarat-Mascle, I. Bloch, D. Vidal-Madjar, Application of DempsterShafer evidence theory to unsupervised classification in multisource remote sensing, IEEE Trans. on Geosci. and Remote Sens. 35 (1997) 1018-1031.

[52] T. Saaty, The analytic hierarchy process, McGraw Hill, New York, 1980.

[53] J. Dezert, J. Tacnet, M. Batton-Hubert, F. Smarandache, Multi-criteria decision making based on DSmT-AHP, in: Int. Conf. on Belief Funct., Brest, France, 2010.

[54] L. Klein, Sensor and Data Fusion: A Tool for Information Assessment and Decision Making, SPIE-The International Society for Optical Engineering, 2004. 
[55] J. Daniel, A. Birouche, J. Lauffenburger, M. Basset, Navigation-based constrained trajectory generation for advanced driver assistance systems, Int. J. of Veh. Auton. Syst. 9 (2011) 269-296.

[56] R. Langley, Dilution of precision, GPS World 10 (1999) 52-59.

[57] S. Durekovic, J. Sixt, S. Dreher, W. Becker, ADASRP 2006 user's manual, Tech. rep., Navigation Technologies (2007).

[58] Navigation Technologies, ADAS Research Platform, Presentation (2002).

[59] F. Moutarde, A. Bargeton, A. Herbin, L. Chanussot, Modular traffic signs recognition applied to on-vehicle real-time visual detection of american and European speed limit signs, in: World Congr. on Int. Trans. Syst., Beijing, China, 2007, pp. $1122-1126$.

[60] Z. Hua, B. Gong, X. Xu, A DS-AHP approach for multi-attribute decision making problem with incomplete information, Expert Syst. with Appl. 34 (2008) 22212227. 